\title{
MATHEMATICAL MODELING OF INFLAMMATORY PROCESSES OF ATHEROSCLEROSIS
}

\author{
G. Abi Younes ${ }^{1}$ And N. El KhatiB ${ }^{2, *}$ (1)
}

\begin{abstract}
Atherosclerosis is a chronic disease which involves the build up of cholesterol and fatty deposits within the inner lining of the artery. It is associated with a progressive thickening and hardening of the arterial wall that result in narrowing of the vessel lumen and restriction of blood flow to vital organs. These events may cause heart attack or stroke, the commonest causes of death worldwide. In this paper we study the early stages of atherosclerosis via a mathematical model of partial differential equations of reaction-diffusion type. The model includes several key species and identifies endothelial hyperpermeability, believed to be a precursor on the onset of atherosclerosis. For simplicity, we reduce the system to a monotone system and provide a biological interpretation for the stability analysis according to endothelial functionality. We investigate as well the existence of solutions of traveling waves type along with numerical simulations. The obtained results are in good agreement with current biological knowledge. Likewise, they confirm and generalize results of mathematical models previously performed in literature. Then, we study the non monotone reduced model and prove the existence of perturbed solutions and perturbed waves, particularly in the bistable case. Finally, we extend the study by considering the complete model proposed initially, perform numerical simulations and provide more specific results. We study the consistency between the reduced and complete model analysis for a certain range of parameters, we elaborate bifurcation diagrams showing the evolution of inflammation upon endothelial permeability and LDL accumulation and we consider the effect of anti-inflammatory process on the system behavior. In this model, the regulation of atherosclerosis progression is mediated by anti-inflammatory responses that, up to certain extent, lead to plaque regression.
\end{abstract}

Mathematics Subject Classification. 35K57, 35C07.

Received October 13, 2021. Accepted January 23, 2022.

\section{Motivation And Biological BACKGRound}

Atherosclerosis is a chronic cardiovascular disease of the arterial wall that involves immunoinflammatory mechanisms as a response to abnormal cholesterol deposits in the inner layers of arteries. The chronic accumulation of fat contributes to the formation of fibrofatty lesions, called atheromatous plaques, resulting in pathologic thickening of the arterial wall. Atherosclerotic lesions develop in the coronary, cerebral, and peripheral arteries and the aorta and restrict the blood flow to vital organs.

Keywords and phrases: Mathematical modeling, atherosclerosis, partial differential equations, traveling waves.

${ }^{1}$ Institut Camille Jordan, UMR 5208 CNRS, University Lyon 1, 69622 Villeurbanne, France.

2 Department of Computer Science and Mathematics, Lebanese American University P.O. Box 36, Byblos, Lebanon.

* Corresponding author: nader.elkhatib@lau.edu.lb 
Clinically, fatty streaks are the earliest pathologic descriptions of atherosclerotic lesions. They evolve into fibrous plaques, some of which develop into advanced plaques aggravated by hemorrhage, ulceration, calcification and thrombosis. The plaques sequentially induce major complications leading to morbidity and mortality worldwide, including myocardial infarctions and strokes, as well as disabling peripheral artery disease [39].

The history of atherosclerosis dates back to ancient times when its early description was considered to be the restriction of the transit of blood through thickening of the vessels. This conveys to the etymology of the term atherosclerosis derived from the Greek words athero, meaning gruel, or paste, corresponding to the accumulation of fatty material in the central core of the plaque, and the term sclerosis, meaning thickening of the intimal layer of arteries [50]. However, recent atherosclerosis literature have profound implications in regarding atherosclerosis as a chronic, low-grade inflammation which is aggravated by hypercholesterolemia and other recognized risk factors [44], including hypertension, cigarette smoking, sedentary activity and diabetes mellitus [47].

Over the past several decades, studies of the pathophysiological process of atherogenesis have provided considerable insight into advanced preventive strategies and enhanced clinical outcomes in affected individuals [39]. But, rather than receding globally, the burden of ischemic cardiovascular conditions has risen to become a top cause of morbidity and mortality worldwide [43]. Thus, on a clinical basis and as a public health challenge, atherosclerosis remains high on the list of global challenges [38].

Continued research promises to provide further progress in combating this common chronic disease [39]. A deeper understanding of molecular mechanisms of atherosclerosis contributes to explore more effective preventive and therapeutic targets.

\section{PAthophysiology of ATherosclerosis}

\subsection{Endothelial dysfunction and activation}

Cardiovascular diseases involve altered size and structure of the arterial wall that typically consists of assembled and patterned layers to provide it with structural integrity and contractility [24].

Some of the large and moderate arteries, such as the aorta and coronary artery, are composed of three layers: the tunica intima that consists of the endothelium, and a basal layer of elastic tissue, the tunica media composed of vascular smooth muscle cells and elastin-rich extracellular matrix and the outermost layer of the vascular wall called the tunica adventitia (see Fig. 1).

The endothelium separates blood from the arterial wall layers and actively regulates the exchange between these compartments. A healthy endothelium, produces a balance of vasoconstrictive and vasodilatory molecules that coordinate vascular tone and can be altered in response to local stimuli [1]. There is a considerable evidence that the vasoprotective effects of the endothelium (vasodilation and inhibition of inflammatory response) are mediated by nitric oxide (NO), a soluble gas, synthesized in endothelial cells (ECs) [54]. Studies have shown that shear stress stimulates the production of NO in endothelium [11]. In addition, NO is involved in proliferation of endothelial cells that maintain normal endothelial functions.

The decreased production or activity of NO causes serious problems to the endothelial equilibrium [54], and any perturbation of the vascular tone balance leads to physiological adaptation or possibly injury of the endothelium.

Endothelial dysfunction comprises a loss of balance between endothelial-derived vasodilatory and vasoconstrictory factors, where the pro-vasoconstrictory state becomes dominant, leading to progressive pathophysiological changes [45]. It is commonly associated with reduced nitric oxide bioavailability [35], and is triggered via a number of different mechanisms, including turbulent blood flow, low shear stress, environmental irritants such as tobacco and hyperlipidaemia [1]. The decreased production or activity of nitric oxide further leads to endothelial cell activation [1], associated with endothelial expression of cell-surface adhesion molecules and endothelial leukocyte adhesion molecule [27]. 


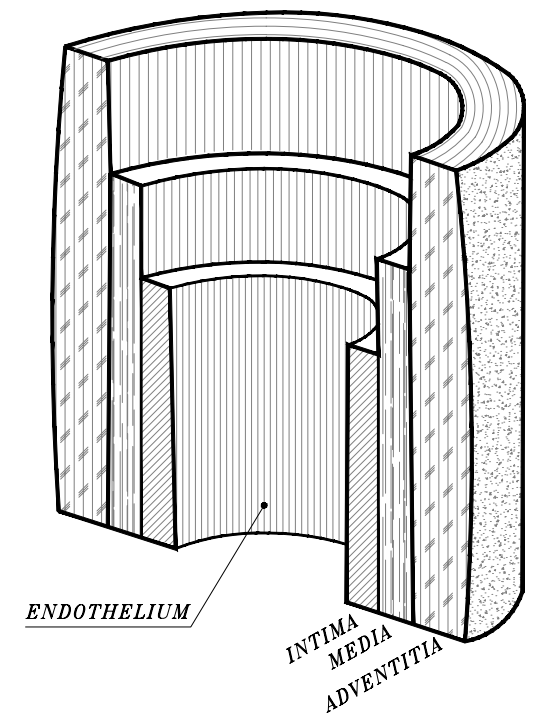

Figure 1. A cross sectional view of the arterial wall that shows three different layers: the intima, the media and the adventitia. The endothelium is a thin layer of cells that lines the inner surface of the artery wall. It forms an interface between the circulating blood in the lumen and the rest of the arterial wall.

Collectively, endothelial dysfunction and endothelial cell activation exhibit pro-inflammatory features [45]: vasoconstriction, loss of vascular integrity causing the efflux of fluids from the intravascular space to the subendothelium, expression of leucocyte adhesion molecules, cytokine production and low density lipoprotein (LDL) oxidation $[27,35]$.

And so, a dysfunctional endothelium promotes intimal retention of cholesterol that subsequently initiates an inflammatory response. Hence, endothelial dysfunction represents an initial step in the development of atherogenesis and is recognized as an early precursor of atherosclerosis [45].

Studies have shown that some factors promote endothelial dysfunction and activation, such as the oxidized LDL in the subendothelial space [34, 36], reactive oxygen species (ROS) [40], low shear stress [53] and some pro-inflammatory cytokines (IL-6 and TNF- $\alpha$ ) [35]. Some other factors have emerged as key regulators of many endothelial cell functions, including barrier function, which are deregulated during atherogenesis, such as high-density lipoproteins (HDLs) [55] and NO [54].

\subsection{Structures involved in endothelial permeability}

Endothelial injury may lead to hyperpermeability, where components of the blood normally confined to the vascular lumen pass through the endothelium, and the rate of such passage is increased [23].

The pathways of plasma constituents such as LDL, HDL, monocytes and T-helper cells across the endothelium is accomplished via transcellular routes (by migrating directly through the body of a single endothelial cell) or paracellular routes (by opening a gap between two adjacent endothelial cells) [12, 22, 46, 55]. In most healthy continuous endothelia, the paracellular pathway is only viable for small solutes.

The first regulator of transendothelial passage is the glycocalyx, a thick matrix layer that lines the inner wall of healthy blood vessels. The endothelial glycocalyx may be damaged by exposure to shear and oxidative stress in conditions of cardiovascular risk factors. The degradation of the endothelial glycocalyx layer reduces endothelial cells production of NO in response to blood shear stress [6]. NO decreased production significantly contributes to the induction of oxidant stress and subsequent impairment of endothelial function by disruption of tight junction integrity resulting in increased permeability [30], as shown in Figure 2. 


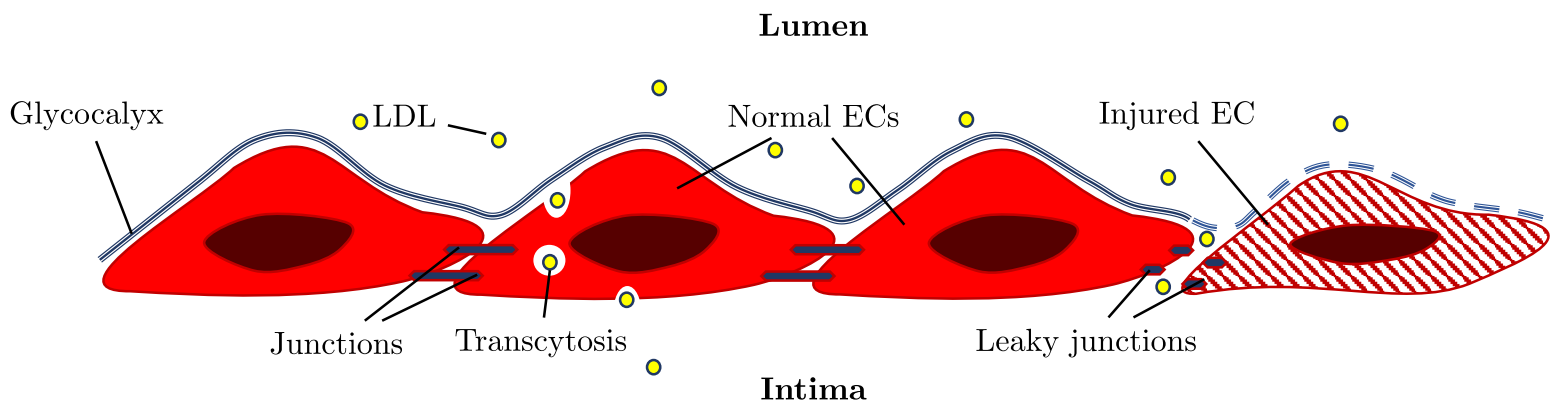

FiguRE 2. Paracellular and transcellular pathways of LDL across the endothelium. Endothelial cell junctions prevent passage of large molecules towards the intima and a healthy glycocalyx regulates the transcytosis of LDL across endothelial cells. However, loss of tight junctions and distortion of the glycocalyx increase penetration of large molecules into subendothelium.

\subsection{Pathogenesis of atherosclerosis}

Endothelial cells are joined together tightly to form a semi-permeable barrier that limits the efflux of large molecules, such as LDL, into the subendothelial spaces [41]. When the endothelium is exposed to cardiovascular risk factors, endothelial dysfunction occurs resulting an impaired vasorelaxation, primarily due to decreased NO bioavailability. Endothelial dysfunction comprises a specific state of endothelial activation [25]. The end-result of endothelial activation and dysfunction may be the loss of microvascular barrier integrity [32], leading to the passage of LDL to the subendothelial space.

Once in the intima, LDL particles undergo a series of chemical modifications that involves oxidation by oxygen free radicals as shown in Figure 3. Oxidized LDL, formed and retained in the subentothelial space, is a harmful type of cholesterol that decreases the availability of endothelial nitric oxide [31], and promotes the activation of endothelial cells through the induction of the cell surface adhesion molecules [41]. Ox-LDL can even induce endothelial cell death [33]. This results in a hyperpermeable endothelium.

However, high density lipoproteins, HDL, mediate an antiatherogenic function at this stage. They inhibit the oxidation of LDL through their direct oxidation. In fact, it has been demonstrated that the lipids of HDL are initially oxidized in preference to those in LDL in vivo [29].

Adhesion molecules secreted by injured endothelial cells, recruits circulating phagocytic white blood cells, such as monocytes, and lymphocytes, such as T cells, to the subendothelium [31]. Monocytes enter lesions and differentiate into macrophages that internalize oxidized lipoproteins. They become engorged with lipids resulting in dysregulated lipid metabolism and a shift in macrophage phenotype to that of lipid-laden foam cells [10]. Foam cells, constitute the primary part of fatty streaks which then proceed with atherosclerosis plaque genesis $[9]$.

Macrophages have been classified as M1/M2 subtypes. M1 macrophages support inflammatory processes that protect the host from microbial infection and aid in the elimination of tumors and M2 macrophages suppress ongoing inflammatory responses, and facilitate tissue repair/remodeling [7]. Both M1 and M2 macrophages have been identified in atherosclerotic plaques. Whereas M2 macrophages are atheroprotective and display antiinflammatory effects, M1 macrophages are heavily involved in atherosclerotic lesion enlargement and progression [52]. Microenvironmental features, such as a variety of pro-inflammatory cytokines, determine the macrophage activation and polarization.

$\mathrm{T}$ cells, a type of leukocytes, have the ability to differentiate into various T-cell subtypes, including $\mathrm{T}$ helper Th1, Th2 and other lineages. There are various factors that may influence lineage phenotypes [5]. They regulate the maturation of monocytes into macrophages [7].

Th1 cells are considered pro-inflammatory through their affiliation with M1 macrophages. They secrete IFN $\gamma$ [5] known to activate M1 macrophages. M1 macrophages promote atherosclerosis progression through secretion of pro-inflammatory cytokines, such as IL-1 $\alpha$, IL-1 $\beta$, IL-6, IL-12, and IL-23 [5, 21], and free radicals, 
to maintain local inflammation [52]. Pro-inflammatory cytokines released by M1 macrophages induce increased vascular permeability and recruitment of inflammatory cells.

Studies have shown that pro-inflammatory cytokines secreted in macrophages are involved in their own production. For instance, IL-1 $\beta$ serves as an inflammatory master cytokine that enhances the expression of many pro-inflammatory cytokines [37]. Moreover, in stimulated macrophages, IL- $1 \alpha$ is synthesized de novo and can be actively secreted [16] in response to a variety of physiological stimuli, including oxidative stress, lipid overload, hormonal stimulation and exposure to cytokines (including IL-1 $\beta$ and IL- $1 \alpha$ itself) [15].

All the same, M2 macrophages are induced by IL-4, IL-5, IL-13, IL-10 and TGF $\beta$ produced by Th2 cells [52]. They produce anti-inflammatory cytokines such as IL-19, IL-27 and IL-33, that stimulate protective responses and suppress atherosclerosis development [20].

The foam cells secrete pro-inflammatory cytokines which recruit additional monocytes and macrophages in a positive feedback mechanism. These foam cells accumulate within the subendothelium giving rise to the plaque. Vascular smooth muscle cells traditionally produce collagen forming the fibrous cap of the lesion and preventing plaque rupture and thrombosis [10].

The foam cells eventually undergo apoptosis and necroptosis. M2 macrophages express anti-inflammatory markers that act to reduce the inflammation of the plaque due to apoptotic and necrotic cells and promote plaque stability. Dead and dying foam cells, if not efficiently cleared by M2 macrophages, become leaky and eventually release their contents into the subendothelium. This process results in the formation of the necrotic or lipid core. As the necrotic core grows and the fibrous cap thins, the plaque is vulnerable to rupture, which may result in acute cardiovascular events such as thrombosis [10].

The balance between proatherosclerotic and atheroprotective immune cells in atherosclerotic plaques is a determining factor in plaque progression and vulnerability. While M1 macrophages have a role in stimulating an inflammatory response, M2 macrophages are responsible for plaque stabilization and plaque regression [52]. Studies on macrophages heterogeneity lead to novel strategies of pharmacological intervention to combat cardiovascular diseases and their complications.

The clinical manifestations of atherosclerosis are the consequences of atherosclerotic plaque rupture or erosion. They comprise myocardial infarction, stroke and aortic aneurysms. Atherosclerotic disease remains the most important cause of death in developed nations, despite advances in medical, interventional, and surgical treatment [13].

\section{Mathematical Modeling OF ATHEROsClerosis IN THE Literature}

Over the recent decades, a broad spectrum of mathematical and computational models of the inflammatory process of atherosclerosis were established using different modeling techniques and strategies. Some consider several key species, where others identify the interaction between blood and the arterial wall. The mechanical factors that could initiate of atherosclerosis lesions have been widely explored by many authors.

The objective of these studies is to pave a way towards more precise understanding of the inflammatory aspect of atherosclerosis and to predict the fate of atherosclerosis. Below is a general overview of some existing models in literature.

Several models inspect the inflammatory aspects of atherosclerosis, considering different species that have a major role in the onset of atherosclerosis. In these works, the inflammation described through the interplay between the species taken into account is the main motive. The basic model studied in [18] considers only two principal species; immune cells and cytokines. Other works consider more detailed biological studies and elaborate a system modeling many other species operating in the establishment of atherosclerosis, as in [8], where the pro and anti-inflammatory processes occurring during atherogenesis are described in detail and in [42], where the cellular mechanisms behind the formation of an atherosclerotic plaque are studied through different stages. Some other works describe the chronic inflammatory response by highlighting the effect of a particular phenomenon contributing to plaque formation. For instance, in [14], the authors focus on the oxidation of LDL particles approach and give a model which represents some of the in vitro experiments carried out on 


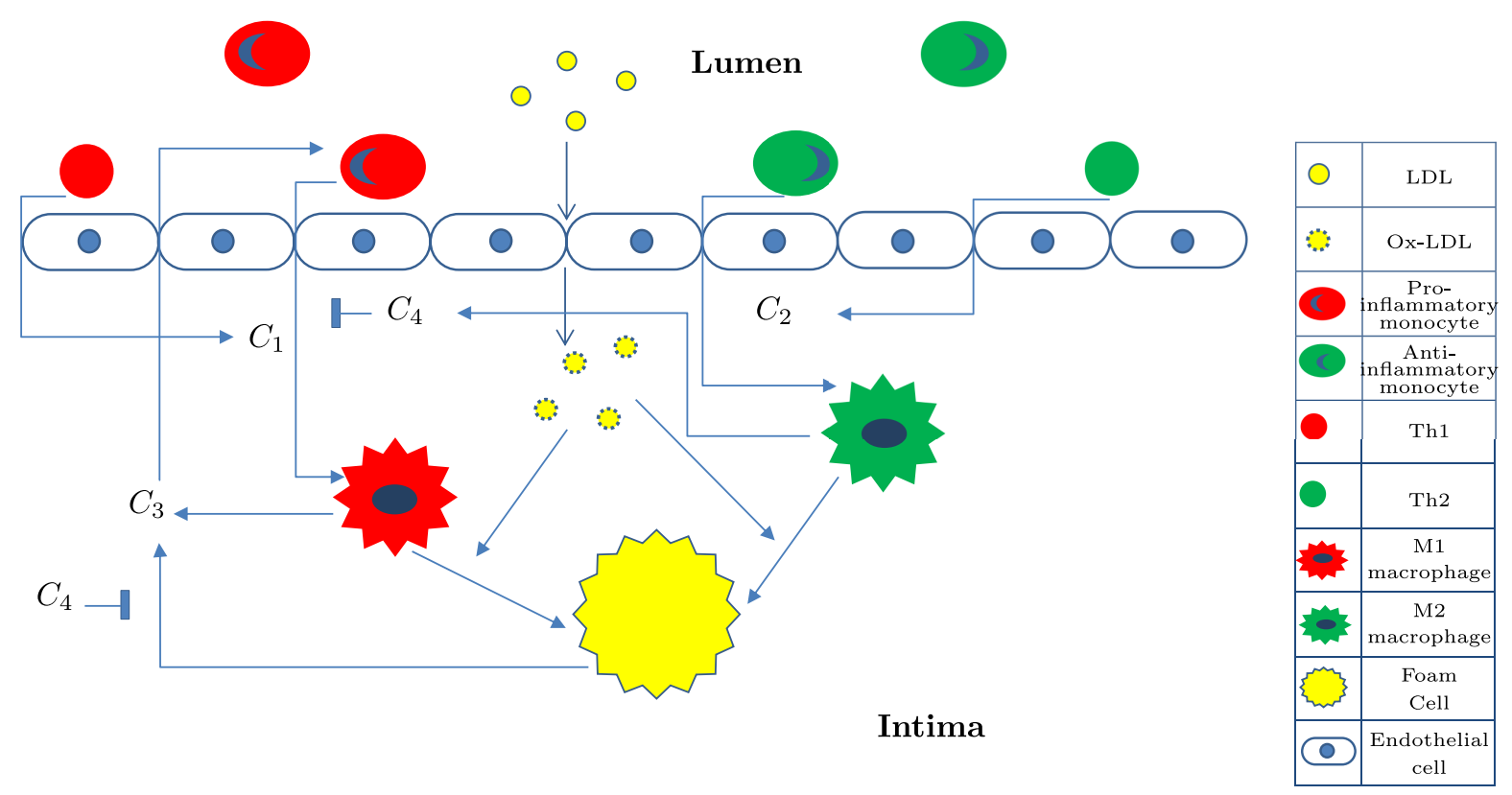

FIGURE 3. Injured endothelial cells increase endothelial permeability allowing LDL particles to reach the intima. LDL particles within the artery walls undergo oxidative and enzymatic modifications, creating ox-LDL. Accumulation of ox-LDL can trigger an inflammatory response inducing the adherence of monocytes to the endothelium and migration into the arterial intima. $\mathrm{T}$ helper cells mediate the differentiation of monocytes into macrophages. Monocytes differentiating into M1 or M2 macrophages favoring either a proatherogenic or atheroprotective profile. The excessive internalization of lipid inside the macrophage results in foamy cells formation.

LDL particles oxidation and their antioxidant defense against free radical attack due to vitamin E particles they possess.

While some mathematical models consider biochemical reactions that initiate and maintain the overall progress of inflammation, some other works examine the contribution of fluid mechanics by investigating the fluid structure interaction and the effects of haemodynamic factors. For instance, in [19], plaque-flow interaction in atherosclerosis is investigated in an FSI model that considers the blood as a viscous fluid and the carotid artery with the atheromatous plaque as hyperelastic materials. In the same manner, in [42], the authors take into account the blood flow to investigate the wall shear stress in cap degradation, as well as the wound healing applied to plaque rupture. Further, the authors in [3] examine complex dynamics associated with plaque growth in human arteries. They use bifurcation analysis to propose a mathematical model. The study considers the interaction between the blood flow and the artery wall combining both blood flow conditions and biochemical evolution of the plaque constituents. It explores the different plaque constituents evolution on short time scales, and their accumulation into the artery on a longer time scale. Coupling the inflammatory processes with the fluid dynamics-mechanical processes was also studied in [51]. This model describes the blood flow, the fluid flow inside the poroelastic vessel wall, and the transport, signaling and interaction processes initiating inflammation and atherosclerosis.

Although endothelial dysfunction or injury is not fully understood, recently, several studies have included endothelial permeability to their models. These studies suggest that passage of key components in atherosclerosis to the intima is due to endothelial permeability. In [51], the authors consider the thickening of the vessel walls and focus on quantifying the functionality of the endothelium. Through this model, the authors describe the diapedesis of monocytes from their transport on the endothelial surface to their influx in the lumen. On the 
other hand, endothelial activation has been investigated in [8] and described as a variable depending on the concentration of ox-LDL and pro-inflammatory cytokines.

Mathematical models usually take the form of systems of either partial differential equations as in $[14,18]$ or ordinary differential equations as in $[8,48]$. The PDEs model normally are of reaction-diffusion type that describe local chemical reactions in which the substances are transformed into each other, and their diffusion. The spatial models are in general one-dimensional as in [18] or two-dimensional as in [17]. Some works consider a one-dimensional model of PDEs then extend the domain into two spatial dimensions in order to generalize the results, as in [42].

For a more prominent description of biological processes, many mathematical models in literature consider complex modeling techniques that result in heavy and complicated analysis. That being so, the authors opt for some simplifications, assumptions or even linearization of the functions, with regard to reducing sophistications and being able to handle the model. Even though these approaches weaken the rigor of the study in terms of illustrating phenomenological processes, the validation of the simplified model results with biological knowledge makes the predicted behavior of the disease through this model reliable within acceptable bounds. These techniques are employed in a large number of models in literature, for example in [48, 49]

Many works in literature, such as $[17,18]$ are devoted to the study of traveling wave solutions for some mathematical models of atherosclerosis of reaction-diffusion equations. The aim of these studies is to describe the propagation of the inflammation through a wave. The existence of traveling wave solutions is ensured due to the monotonicity of the systems. Whereas, in [49], the authors not only consider the wave patterns in reaction diffusion systems but also they discuss the dynamics of wave patterns underlying cardiac arrhythmias that may lead to clot formation or stroke.

Mathematical models of atherosclerosis are effective tools used successfully in understanding fundamental processes in atherosclerosis. They have been studied with the objective of obtaining insights on inflammation. They essentially provide simulations detecting system behaviors and featuring the evolution of the disease. Collectively, numerical simulations and analytical data reported from biological studies are an attempt to quantify the inflammatory process and to afford predictive results. Several models provide numerical simulations and rely on existing data to convey to reliable predictive conclusions. One can list the following works: [17-19, $28,49,51]$.

Mathematical models of atherosclerosis support current concepts about the inflammation and can be useful tools to quantitatively evaluate the evolution of the disease. They afford a better understanding of different aspects of atherosclerosis. For example, the study in [18] considers a critical parameter that affected the initiation of the disease represents the concentration of ox-LDL in the intima. The mathematical study of the kinetic system leads in the following biological interpretation: low ox-LDL concentrations do not trigger any chronic inflammatory reaction ; intermediate concentration of ox-LDL leads to a bistable case where a chronic inflammatory reaction can set up when the system overcomes a certain threshold ; and high ox-LDL concentrations correspond to a monostable system where even a small perturbation of the non inflammatory state leads to the setup of a chronic inflammatory response. Moreover, the authors in [19] conclude that recirculation of blood leads to clot formation and that the vessel wall movement due to the stress over the plaque is implicated in the plaque vulnerability. Coupled 0D-1D models of blood flow in atherosclerotic vasculature analyzed in this paper provide a basis for patient-specific analysis in surgery treatments of atherosclerosis. Further, paper [3] distinguishes the most relevant factors to the plaque formation. Besides, in [48], the model shows that it is macrophage proliferation that drives lesion instability rather than an increasing influx of modified LDL. In other words, many individuals develop lesions despite having a normal lipoprotein profile. Likewise, the authors in [14] conclude that HDL could provide a protection for LDL against radical attack through the reverse cholesterol transport mechanism and that vitamin E supplementation is not as beneficial as HDLs in reducing LDLs oxidation. Numerical simulations in [28] lead to some results that are in agreement with cardiovascular disease features such as the localization of immune cells, the build-up of lipids and debris and the formation of a cap of smooth muscle cells. The results of paper [2] lead to the conclusion that hypertension greatly increases the transmural filtration and concentration polarization at the lumen/endothelium interface and that geometrical 
TABLE 1. Notations description.

\begin{tabular}{cl}
\hline$L$ & Concentration of LDL \\
$H$ & Concentration of HDL \\
$L_{o x}$ & Concentration of ox-LDL \\
$A_{1}$ & Concentration of pro-inflammatory monocytes \\
$A_{2}$ & Concentration of anti-inflammatory monocytes \\
$M_{1}$ & Concentration of pro-inflammatory (M1) macrophages \\
$M_{2}$ & Concentration of anti-inflammatory (M2) macrophages \\
$T_{1}$ & Concentration of Th1 \\
$T_{2}$ & Concentration of Th2 \\
$C_{1}$ & Concentration of Th1 cytokines \\
$C_{2}$ & Concentration of Th2 cytokines \\
$C_{3}$ & Concentration of pro-inflammatory cytokines produced by M1 macrophages \\
$C_{4}$ & Concentration of anti-inflammatory cytokines produced by M2 macrophages \\
$F$ & Concentration of foam cells \\
\hline
\end{tabular}

variation on the LDL accumulation within the wall is greatly increased near the region of stenosis. The dynamical model of lipoprotein metabolism introduced in [4] shows bistability and hysteresis between a low and a high cholesterol state. The bifurcation analysis can be related to diet and statin medication and reverse cholesterol transport through HDL. Sensitivity analysis proves that the most robust feature in the low cholesterol state is the concentration of intracellular cholesterol, while the plasma concentrations can vary widely. Estimates for the characteristic time scales governing the dynamics of the model in the low and high cholesterol states are established. And finally, the study in paper [51] provides results on dynamics of endothelial permeability, as well as the growth and spread of immune cells populations and their dependence in particular on LDL and wall-shear stress.

\section{MATHEMATiCAL MODEL}

In this section, we model the different cascades of atherogenesis in a system of coupled partial differential equations. We consider some key-role players in the inflammatory process shown in Table 1. The endothelial dysfunction and activation are taken into consideration through a term $P$ considered to be an endothelial permeability indicator. The modeling of the term $\mathrm{P}$ can involve the effect of ox-LDL and free radicals in endothelial dysfunction, the effect of pro-inflammatory cytokines in endothelial activation, the effect of HDL in preserving endothelium and finally, the effect of nitric oxide or shear stress.

For $l>0, x \in] 0, l[$ and $t \in[0, \infty[$, the system reads as follows:

The following equation models the evolution of LDL concentration:

$$
\frac{\partial L}{\partial t}=D_{1} \frac{\partial^{2} L}{\partial x^{2}}+\sigma_{L} P L_{0}-k_{L} L
$$

The first right-hand side term in equation (4.1) represents the diffusion of LDL and the second right-hand side term describes the LDL penetration through the vessel wall, where $L_{0}$ is the concentration of LDL in the blood. The third right-hand side term describes the oxidization of LDL particles.

In subsequent equations, the terms $D_{i}$, for $i=2, \ldots, 14$, refer to the diffusion rates.

Similar reactions are modeled for the HDL concentration as follows:

$$
\frac{\partial H}{\partial t}=D_{2} \frac{\partial^{2} H}{\partial x^{2}}+\sigma_{H} P H_{0}-k_{H} H
$$


The HDL penetration through the vessel wall is described through the second right-hand side term in equation (4.2), where $H_{0}$ is the concentration of HDL in the blood. The consumption of HDL through oxidation reaction with free radicals is represented in the third right-hand side term.

The evolution of ox-LDL concentration in the intima is modeled by the following equation:

$$
\frac{\partial L_{o x}}{\partial t}=D_{3} \frac{\partial^{2} L_{o x}}{\partial x^{2}}+k_{L} L-\lambda_{L_{o x} M_{1}} \frac{L_{o x}}{k_{L_{o x}}+L_{o x}} M_{1}-\lambda_{L_{o x} M_{2}} \frac{L_{o x}}{k_{L_{o x}}+L_{o x}} M_{2}-d_{L_{o x}} L_{o x}
$$

We model the production of ox-LDL due to LDL oxidation by reaction with the radicals through the term $k_{L} L$ in equation (4.3), and the ingestion of ox-LDL by M1 and M2 macrophages through the third and fourth right-hand side terms. The last term models the degradation of ox-LDL.

The description of monocytes concentrations evolution is given by:

$$
\begin{gathered}
\frac{\partial A_{1}}{\partial t}=D_{4} \frac{\partial^{2} A_{1}}{\partial x^{2}}+\lambda_{P A_{1}} P A_{1}^{0}-\lambda_{C_{1} A_{1}} \frac{C_{1}}{k_{C_{1}}+C_{1}+k_{4} C_{4}} A_{1}-d_{A_{1}} A_{1}, \\
\frac{\partial A_{2}}{\partial t}=D_{5} \frac{\partial^{2} A_{2}}{\partial x^{2}}+\lambda_{P A_{2}} P A_{2}^{0}-\lambda_{C_{2} A_{2}} \frac{C_{2}}{k_{C_{2}}+C_{2}} A_{2}-d_{A_{2}} A_{2} .
\end{gathered}
$$

The terms $\lambda_{P A_{1}} P A_{1}^{0}$ and $\lambda_{P A_{2}} P A_{2}^{0}$, in equations (4.4) and (4.5), model the $A_{1}$ and $A_{2}$ monocytes penetration through the vessel wall, where $A_{1}^{0}$ and $A_{2}^{0}$ are densities of $A_{1}$ and $A_{2}$ monocytes in blood. The third right-hand side terms describe the differentiation of monocytes into macrophages, and the last right-hand side terms correspond to the death of monocytes.

The following equations describe the evolution of M1 and M2 macrophages concentrations:

$$
\begin{gathered}
\frac{\partial M_{1}}{\partial t}=D_{6} \frac{\partial^{2} M_{1}}{\partial x^{2}}+\lambda_{C_{1} A_{1}} \frac{C_{1}}{k_{C_{1}}+C_{1}+k_{4} C_{4}} A_{1}-\lambda_{L_{o x} M_{1}} \frac{L_{o x}}{k_{L_{o x}}+L_{o x}} M_{1}-d_{M_{1}} M_{1}, \\
\frac{\partial M_{2}}{\partial t}=D_{7} \frac{\partial^{2} M_{2}}{\partial x^{2}}+\lambda_{C_{2} A_{2}} \frac{C_{2}}{k_{C_{2}}+C_{2}} A_{2}-\lambda_{L_{o x} M_{2}} \frac{L_{o x}}{k_{L_{o x}}+L_{o x}} M_{2}-d_{M_{2}} M_{2} .
\end{gathered}
$$

The second right-hand side terms of equations (4.6) and (4.7) correspond to the differentiation of monocytes into macrophages, the third right-hand side terms describe the uptake of ox-LDL by macrophages and the transformation of macrophages into foam cells, and the fourth right-hand side terms represent the death of macrophages.

The intimal concentrations of T-helper cells satisfy the following equations:

$$
\begin{aligned}
& \frac{\partial T_{1}}{\partial t}=D_{8} \frac{\partial^{2} T_{1}}{\partial x^{2}}+\lambda_{P T_{1}} P T_{1}^{0}-d_{T_{1}} T_{1}, \\
& \frac{\partial T_{2}}{\partial t}=D_{9} \frac{\partial^{2} T_{2}}{\partial x^{2}}+\lambda_{P T_{2}} P T_{2}^{0}-d_{T_{2}} T_{2} .
\end{aligned}
$$

Equations (4.8) and (4.9) model the influx of T-helper cells from the blood into the intima due to the permeability of the endothelium and the death of T-helper cells. 
The concentrations of cytokines released by T-helper cells are described through the following equations:

$$
\begin{gathered}
\frac{\partial C_{1}}{\partial t}=D_{10} \frac{\partial^{2} C_{1}}{\partial x^{2}}+\lambda_{C_{1}} T_{1}-\lambda_{C_{1} A_{1}} \frac{C_{1}}{k_{C_{1}}+C_{1}+k_{4} C_{4}} A_{1}-d_{C_{1}} C_{1}, \\
\frac{\partial C_{2}}{\partial t}=D_{11} \frac{\partial^{2} C_{2}}{\partial x^{2}}+\lambda_{C_{2}} T_{2}-\lambda_{C_{2} A_{2}} \frac{C_{2}}{k_{C_{2}}+C_{2}} A_{2}-d_{C_{2}} C_{2} .
\end{gathered}
$$

We model the production of $C_{1}$ and $C_{2}$ cytokines by T-cells in equations (4.10) and (4.11) at rates of $\lambda_{C_{1}}$ and $\lambda_{C_{2}}$. The differentiation of $A_{1}$ into $M_{1}$ macrophages (down regulated by $C_{4}$ ) and the differentiation of $A_{2}$ monocytes into $M_{2}$ macrophages are modeled by the third right-hand side terms. The last terms correspond to the degradation of cytokines.

In the following equations, we model the evolution in time of cytokines produced by M1 and M2 macrophages:

$$
\frac{\partial C_{3}}{\partial t}=D_{12} \frac{\partial^{2} C_{3}}{\partial x^{2}}+\lambda_{C_{3} M_{1}} \frac{C_{3}}{k_{C_{3}}+C_{3}+k C_{4}} M_{1}+\lambda_{C_{4} F} \frac{1}{k_{C_{4}}+C_{4}} F-d_{C_{3}} C_{3} .
$$

The second right-hand side term in equation (4.12) describes the production of pro-inflammatory cytokines due to the presence of macrophages and the pro-inflammatory cytokines themselves. This production is inhibited by the anti-inflammatory agents $C_{4}$. The third right-hand side term models the secretion of pro-inflammatory cytokines by the foam cells, regulated by $C_{4}$, and the last term refers to the degradation of pro-inflammatory cytokines.

$$
\frac{\partial C_{4}}{\partial t}=D_{13} \frac{\partial^{2} C_{4}}{\partial x^{2}}+\lambda_{C_{4}} M_{2}-d_{C_{4}} C_{4} .
$$

The production of anti-inflammatory cytokines $C_{4}$ by $M_{2}$ macrophages is described by the second right-hand side term in equation (4.13). The third right-hand side term corresponds to the degradation of cytokines.

The evolution of foam cells concentration is modeled as follows:

$$
\frac{\partial F}{\partial t}=D_{14} \frac{\partial^{2} F}{\partial x^{2}}+\lambda_{L_{o x} M_{1}} \frac{L_{o x}}{k_{L_{o x}}+L_{o x}} M_{1}+\lambda_{L_{o x} M_{2}} \frac{L_{o x}}{k_{L_{o x}}+L_{o x}} M_{2}-d_{F} F .
$$

The second and third right-hand side terms of equation (4.14) correspond to the formation of foam cells due to the ingestion of ox-LDL by $M_{1}$ and $M_{2}$ macrophages. The last term describes the death of foam cells.

\subsection{Reduced model}

In this section, we consider a reduced model that originates from the complete model (4.1)-(4.14). In this reduced model, we particularly consider the pro-inflammatory process. Therefore, we let $H=A_{2}=M_{2}=$ $T_{2}=C_{2}=C_{4}=F=0$. We further consider the production of Th1 cells and Th1 cytokines are compensated respectively by the loss of Th1 cells and Th1 cytokines, in an unbiased manner. For the sake of ease, we also take $d_{A_{1}}=k_{C_{1}}=0$.

We consider the following new notations: $\lambda_{1}=\sigma_{L} L_{0}, \lambda_{2}=\lambda_{L_{o x} M_{1}}, \lambda_{3}=\lambda_{P A_{1}} A_{1}^{0}, \lambda_{4}=\lambda_{C_{1} A_{1}}, \lambda_{5}=\lambda_{C_{3} M_{1}}$, $k_{2}=k_{L_{o x}}, k_{3}=k_{C_{3}}, d_{1}=d_{L_{o x}}, d_{2}=d_{M_{1}}$ and $d_{3}=d_{C_{3}}$. The reduced model becomes:

$$
\frac{\partial L}{\partial t}=D_{1} \frac{\partial^{2} L}{\partial x^{2}}+\lambda_{1} P-k_{L} L
$$




$$
\begin{gathered}
\frac{\partial L_{o x}}{\partial t}=D_{3} \frac{\partial^{2} L_{o x}}{\partial x^{2}}+k_{L} L-\lambda_{2} \frac{L_{o x}}{k_{2}+L_{o x}} M_{1}-d_{1} L_{o x}, \\
\frac{\partial A_{1}}{\partial t}=D_{4} \frac{\partial^{2} A_{1}}{\partial x^{2}}+\lambda_{3} P-\lambda_{4} A_{1}, \\
\frac{\partial M_{1}}{\partial t}=D_{6} \frac{\partial^{2} M_{1}}{\partial x^{2}}+\lambda_{4} A_{1}-\lambda_{2} \frac{L_{o x}}{k_{2}+L_{o x}} M_{1}-d_{2} M_{1}, \\
\frac{\partial C_{3}}{\partial t}=D_{12} \frac{\partial^{2} C_{3}}{\partial x^{2}}+\lambda_{5} \frac{C_{3}}{k_{3}+C_{3}} M_{1}-d_{3} C_{3} .
\end{gathered}
$$

In the ensuing sections, we study this reduced model with a constant permeability indicator $P$, and with a variable one.

\subsubsection{Reduced model with variable $P$}

In this section, we consider the term $P$ to be a function of $L_{o x}$ and $C_{3}$ as follows:

$$
P=H(\alpha) \frac{P_{0}+L_{o x}+c C_{3}}{k_{1}+L_{o x}+c C_{3}}
$$

where:

- $\alpha$ refers to the assessment of endothelial dysfunction due to external stimuli. Note that vascular function can be measured by several methods, including invasive and non-invasive techniques. $\alpha$ is an independant parameter that is altered upon exposure of the endothelium to cardiovascular risk factors such as low shear stress, nicotine circulating in blood and high plasma concentration of cholesterol.

- $H(\alpha)$ describes the activation and dysfunction of the endothelium and is given by

$$
H(\alpha)= \begin{cases}0 & \text { if } \alpha<\alpha_{0} \\ 1 & \text { otherwise }\end{cases}
$$

where $\alpha_{0}$ represents a threshold to discriminate between normal and abnormal endothelial function. A thick glycocalyx, active endothelial cells, an appropriate endothelial production of NO and a tight chain of junctions are typical features of a normal endothelium. An endothelium in good condition is then assessed through the parameter $\alpha$ with $\alpha<\alpha_{0}$. When the glycocalyx is damaged by cardiovascular risk factors conditions, it causes a decreased production of NO, that in turn results in endothelial dysfunction and activation and tight junctions disruption. All the cascades together lead to increased endothelial permeability. Thus, it is the degradation of the glycocalyx that initiates endothelial dysfunction. Impaired endothelial function associated with a compromised glycocalyx is detected when $\alpha>\alpha_{0}$.

- The term $\frac{P_{0}+L_{o x}+c C_{3}}{k_{1}+L_{o x}+c C_{3}}$ describes the permeability of the endothelium, where $P_{0}$ refers to the selective permeability of a healthy endothelium, i.e. the permeability to small molecules and $k_{1}$ denotes the effect of endothelial regulators, i.e. HDL, NO and SS. 
We substitute the following variables $\tilde{C_{3}}=c C_{3}, \tilde{k_{3}}=c k_{3}, \tilde{\lambda_{5}}=c \lambda_{5}$ and $\tilde{D_{12}}=\frac{D_{12}}{c}$, in equations (4.15)(4.19), and use $P$ as in equation (4.20), then we drop the tildes. The new reduced system reads:

$$
\begin{gathered}
\frac{\partial L}{\partial t}=D_{1} \frac{\partial^{2} L}{\partial x^{2}}+\lambda_{1} H(\alpha) \frac{P_{0}+L_{o x}+C_{3}}{k_{1}+L_{o x}+C_{3}}-k_{L} L, \\
\frac{\partial L_{o x}}{\partial t}=D_{3} \frac{\partial^{2} L_{o x}}{\partial x^{2}}+k_{L} L-\lambda_{2} \frac{L_{o x}}{k_{2}+L_{o x}} M_{1}-d_{1} L_{o x}, \\
\frac{\partial A_{1}}{\partial t}=D_{4} \frac{\partial^{2} A_{1}}{\partial x^{2}}+\lambda_{3} H(\alpha) \frac{P_{0}+L_{o x}+C_{3}}{k_{1}+L_{o x}+C_{3}}-\lambda_{4} A_{1}, \\
\frac{\partial M_{1}}{\partial t}=D_{6} \frac{\partial^{2} M_{1}}{\partial x^{2}}+\lambda_{4} A_{1}-\lambda_{2} \frac{L_{o x}}{k_{2}+L_{o x}} M_{1}-d_{2} M_{1}, \\
\frac{\partial C_{3}}{\partial t}=D_{12} \frac{\partial^{2} C_{3}}{\partial x^{2}}+\lambda_{5} \frac{C_{3}}{k_{3}+C_{3}} M_{1}-d_{3} C_{3} .
\end{gathered}
$$

The kinetic system becomes:

$$
\begin{gathered}
\frac{\mathrm{d} L}{\mathrm{~d} t}=\lambda_{1} H(\alpha) \frac{P_{0}+L_{o x}+C_{3}}{k_{1}+L_{o x}+C_{3}}-k_{L} L, \\
\frac{d L_{o x}}{\mathrm{~d} t}=k_{L} L-\lambda_{2} \frac{L_{o x}}{k_{2}+L_{o x}} M_{1}-d_{1} L_{o x}, \\
\frac{\mathrm{d} A_{1}}{\mathrm{~d} t}=\lambda_{3} H(\alpha) \frac{P_{0}+L_{o x}+C_{3}}{k_{1}+L_{o x}+C_{3}}-\lambda_{4} A_{1}, \\
\frac{\mathrm{d} M_{1}}{\mathrm{~d} t}=\lambda_{4} A_{1}-\lambda_{2} \frac{L_{o x}}{k_{2}+L_{o x}} M_{1}-d_{2} M_{1}, \\
\frac{\mathrm{d} C_{3}}{\mathrm{~d} t}=\lambda_{5} \frac{C_{3}}{k_{3}+C_{3}} M_{1}-d_{3} C_{3} .
\end{gathered}
$$

The Jacobian matrix of system (4.22)-(4.26) reads: 


$$
J=\left[\begin{array}{ccccc}
-k_{L} & \lambda_{1} H(\alpha) \frac{k_{1}-P_{0}}{\left(k_{1}+L_{o x}+C_{3}\right)^{2}} & 0 & 0 & \lambda_{1} H(\alpha) \frac{k_{1}-P_{0}}{\left(k_{1}+L_{o x}+C_{3}\right)^{2}} \\
k_{L} & \lambda_{2} \frac{k_{2} M_{1}}{\left(k_{2}+L_{o x}\right)^{2}}-d_{1} & 0 & -\lambda_{2} \frac{L_{o x}}{k_{2}+L_{o x}} & 0 \\
0 & \lambda_{3} H(\alpha) \frac{k_{1}-P_{0}}{\left(k_{1}+L_{o x}+C_{3}\right)^{2}} & -\lambda_{4} & 0 & \lambda_{3} H(\alpha) \frac{k_{1}-P_{0}}{\left(k_{1}+L_{o x}+C_{3}\right)^{2}} \\
0 & -\lambda_{2} \frac{k_{2} M_{1}}{\left(k_{2}+L_{o x}\right)^{2}} & \lambda_{4} & -\lambda_{2} \frac{L_{o x}}{k_{2}+L_{o x}}-d_{2} & 0 \\
0 & 0 & 0 & \lambda_{5} \frac{C_{3}}{k_{3}+C_{3}} & \lambda_{5} \frac{k_{3} M_{1}}{\left(k_{3}+C_{3}\right)^{2}}-d_{3}
\end{array}\right] .
$$

We recall that a system is said to be monotone if the off-diagonal elements of its Jacobian are non negative. To ensure the monotonicity of system (4.22)-(4.26), we assume that $k_{1}>P_{0}$, and we conduct the study for $\lambda_{2}=0$, then we can extend the results for $\lambda_{2}$ close to zero, by applying the implicit function theorem for the existence and stability of the fixed points.

For the sake of ease, we take $P_{0}=0$. Similarly, we use the implicit function theorem to conclude for $P_{0}$ in a small positive neighborhood of 0 .

Therefore, finding the fixed points of system (4.27)-(4.31) with $\lambda_{2}=P_{0}=0$ amounts to solving the following system:

$$
\begin{gathered}
\lambda_{1} H(\alpha) \frac{L_{o x}+C_{3}}{k_{1}+L_{o x}+C_{3}}-d_{1} L_{o x}=0, \\
\lambda_{3} H(\alpha) \frac{L_{o x}+C_{3}}{k_{1}+L_{o x}+C_{3}}-d_{2} M_{1}=0, \\
\lambda_{5} \frac{C_{3}}{k_{3}+C_{3}} M_{1}-d_{3} C_{3}=0, \\
L=\frac{\lambda_{1}}{k_{L}} H(\alpha) \frac{L_{o x}+C_{3}}{k_{1}+L_{o x}+C_{3}}, \\
A_{1}=\frac{\lambda_{3}}{\lambda_{4}} H(\alpha) \frac{L_{o x}+C_{3}}{k_{1}+L_{o x}+C_{3}} .
\end{gathered}
$$

Since the unknowns of system (4.22)-(4.26) represent concentrations of physical quantities, we hereinafter consider only real nonnegative solutions.

Fixed points: In order to investigate the solutions of system (4.27)-(4.31), we start by examining the case where $\alpha<\alpha_{0}$. Clearly, the unique solution is $E_{1}=(0,0,0,0,0)$.

As for $\alpha>\alpha_{0}$, we proceed as follows:

Equation (4.34) leads to two different solutions: $C_{3}=0$ or $C_{3}=\frac{\lambda_{5}}{d_{3}} M_{1}-k_{3}$. 
We notice that solving equations (4.32) and (4.33) for $C_{3}=0$ is equivalent to solve

$$
d_{1} L_{o x}^{2}+\left(d_{1} k_{1}-\lambda_{1}\right) L_{o x}=0 .
$$

The latter equation admits two solutions:

$$
L_{o x_{0}}=\frac{\lambda_{1}-d_{1} k_{1}-\sqrt{\left(\lambda_{1}-d_{1} k_{1}\right)^{2}}}{2 d_{1}} \text { and } L_{o x_{1}}=\frac{\lambda_{1}-d_{1} k_{1}+\sqrt{\left(\lambda_{1}-d_{1} k_{1}\right)^{2}}}{2 d_{1}} .
$$

Therefore, in the hyperplane $C_{3}=0$, there exists two fixed points $E_{0}$ and $E_{1}$ having the following components:

$$
\begin{aligned}
E_{0} & =\left(\frac{\lambda_{1}}{k_{L}} \frac{L_{o x_{0}}}{k_{1}+L_{o x_{0}}}, L_{o x_{0}}, \frac{\lambda_{3}}{\lambda_{4}} \frac{L_{o x_{0}}}{k_{1}+L_{o x_{0}}}, \frac{d_{1} \lambda_{3}}{d_{2} \lambda_{1}} L_{o x_{0}}, 0\right) \\
& = \begin{cases}\left(\frac{\lambda_{1}-d_{1} k_{1}}{k_{L}}, \frac{\lambda_{1}-d_{1} k_{1}}{d_{1}}, \frac{\lambda_{3}}{\lambda_{4}} \frac{\lambda_{1}-d_{1} k_{1}}{\lambda_{1}}, \frac{\lambda_{3}}{d_{2} \lambda_{1}}\left(\lambda_{1}-d_{1} k_{1}\right), 0\right) & \text { if } \lambda_{1}<d_{1} k_{1}, \\
(0,0,0,0,0) & \text { if } \lambda_{1}>d_{1} k_{1} .\end{cases} \\
E_{1} & =\left(\frac{\lambda_{1}}{k_{L}} \frac{L_{o x_{1}}}{k_{1}+L_{o x_{1}}}, L_{o x_{1}}, \frac{\lambda_{3}}{\lambda_{4}} \frac{L_{o x_{1}}}{k_{1}+L_{o x_{1}}}, \frac{d_{1} \lambda_{3}}{d_{2} \lambda_{1}} L_{o x_{1}}, 0\right) \\
& = \begin{cases}(0,0,0,0,0) & \text { if } \lambda_{1}<d_{1} k_{1}, \\
\left(\frac{\lambda_{1}-d_{1} k_{1}}{k_{L}}, \frac{\lambda_{1}-d_{1} k_{1}}{d_{1}}, \frac{\lambda_{3}}{\lambda_{4}} \frac{\lambda_{1}-d_{1} k_{1}}{\lambda_{1}}, \frac{\lambda_{3}}{d_{2} \lambda_{1}}\left(\lambda_{1}-d_{1} k_{1}\right), 0\right) & \text { if } \lambda_{1}>d_{1} k_{1} .\end{cases}
\end{aligned}
$$

In a similar manner, we proceed in the plane $C_{3}=\frac{\lambda_{5}}{d_{3}} M_{1}-k_{3}$. The $L_{o x}$ component of the fixed points in this plane verify the following equation, that is obtained from equations (4.32) and (4.33):

$$
-d_{1}\left(1+\frac{d_{1} \lambda_{3} \lambda_{5}}{d_{2} d_{3} \lambda_{1}}\right) L_{o x}^{2}+\left(\lambda_{1}\left(1+\frac{d_{1} \lambda_{3} \lambda_{5}}{d_{2} d_{3} \lambda_{1}}\right)-d_{1}\left(k_{1}-k_{3}\right)\right) L_{o x}-k_{3} \lambda_{1}=0 .
$$

Equation (4.38) admits two solutions:

$$
\begin{gathered}
L_{o x_{u}}=\frac{\lambda_{1}\left(1+\frac{d_{1} \lambda_{3} \lambda_{5}}{d_{2} d_{3} \lambda_{1}}\right)-d_{1}\left(k_{1}-k_{3}\right)-\sqrt{\left(\lambda_{1}\left(1+\frac{d_{1} \lambda_{3} \lambda_{5}}{d_{2} d_{3} \lambda_{1}}\right)-d_{1}\left(k_{1}-k_{3}\right)\right)^{2}-4 d_{1} k_{3} \lambda_{1}\left(1+\frac{d_{1} \lambda_{3} \lambda_{5}}{d_{2} d_{3} \lambda_{1}}\right)}}{2 d_{1}\left(1+\frac{d_{1} \lambda_{3} \lambda_{5}}{d_{2} d_{3} \lambda_{1}}\right)}, \text { and } \\
L_{o x_{2}}=\frac{\lambda_{1}\left(1+\frac{d_{1} \lambda_{3} \lambda_{5}}{d_{2} d_{3} \lambda_{1}}\right)-d_{1}\left(k_{1}-k_{3}\right)+\sqrt{\left(\lambda_{1}\left(1+\frac{d_{1} \lambda_{3} \lambda_{5}}{d_{2} d_{3} \lambda_{1}}\right)-d_{1}\left(k_{1}-k_{3}\right)\right)^{2}-4 d_{1} k_{3} \lambda_{1}\left(1+\frac{d_{1} \lambda_{3} \lambda_{5}}{d_{2} d_{3} \lambda_{1}}\right)}}{2 d_{1}\left(1+\frac{d_{1} \lambda_{3} \lambda_{5}}{d_{2} d_{3} \lambda_{1}}\right)} .
\end{gathered}
$$

Upon that, we consider the following inequalities:

- Condition A:

$$
\left(\lambda_{1}\left(1+\frac{d_{1} \lambda_{3} \lambda_{5}}{d_{2} d_{3} \lambda_{1}}\right)-d_{1}\left(k_{1}-k_{3}\right)\right)^{2}-4 d_{1} k_{3} \lambda_{1}\left(1+\frac{d_{1} \lambda_{3} \lambda_{5}}{d_{2} d_{3} \lambda_{1}}\right)>0 .
$$


- Condition B:

$$
\lambda_{1}\left(1+\frac{d_{1} \lambda_{3} \lambda_{5}}{d_{2} d_{3} \lambda_{1}}\right)-d_{1}\left(k_{1}-k_{3}\right)>0 .
$$

- Condition C:

$$
\frac{d_{1} \lambda_{3} \lambda_{5}}{d_{2} d_{3} \lambda_{1}} L_{o x_{2}}-k_{3}>0
$$

For simplicity, when conditions $\mathrm{A}$ and $\mathrm{B}$ are satisfied and $k_{1}<k_{3}$, we assume that:

$$
-\lambda_{1}\left(1+\frac{d_{1} \lambda_{3} \lambda_{5}}{d_{2} d_{3} \lambda_{1}}\right)-d_{1}\left(k_{1}-k_{3}\right)+\sqrt{\left(\lambda_{1}\left(1+\frac{d_{1} \lambda_{3} \lambda_{5}}{d_{2} d_{3} \lambda_{1}}\right)-d_{1}\left(k_{1}-k_{3}\right)\right)^{2}-4 d_{1} k_{3} \lambda_{1}\left(1+\frac{d_{1} \lambda_{3} \lambda_{5}}{d_{2} d_{3} \lambda_{1}}\right)}<0 .
$$

In case that conditions $\mathrm{A}, \mathrm{B}$ and $\mathrm{C}$ are verified, there exists two fixed points $E_{u}$ and $E_{2}$ in the plane $C_{3}=\frac{\lambda_{4}}{d_{3}} M_{1}-k_{3}$, where:

$E_{u}=\left(\frac{\lambda_{1}}{k_{L}} \frac{L_{o x_{u}}+\frac{d_{1} \lambda_{3} \lambda_{5}}{d_{2} d_{3} \lambda_{1}} L_{o x_{u}}-k_{3}}{k_{1}+L_{o x_{u}}+\frac{d_{1} \lambda_{3} \lambda_{5}}{d_{2} d_{3} \lambda_{1}} L_{o x_{u}}-k_{3}}, L_{o x_{u}}, \frac{\lambda_{3}}{\lambda_{4}} \frac{L_{o x_{u}}+\frac{d_{1} \lambda_{3} \lambda_{5}}{d_{2} d_{3} \lambda_{1}} L_{o x_{u}}-k_{3}}{k_{1}+L_{o x_{u}}+\frac{d_{1} \lambda_{3} \lambda_{5}}{d_{2} d_{3} \lambda_{1}} L_{o x_{u}}-k_{3}}, \frac{d_{1} \lambda_{3}}{d_{2} \lambda_{1}} L_{o x_{u}}, \frac{d_{1} \lambda_{3} \lambda_{5}}{d_{2} d_{3} \lambda_{1}} L_{o x_{u}}-k_{3}\right)$,

and

$$
E_{2}=\left(\frac{\lambda_{1}}{k_{L}} \frac{L_{o x_{2}}+\frac{d_{1} \lambda_{3} \lambda_{5}}{d_{2} d_{3} \lambda_{1}} L_{o x_{2}}-k_{3}}{k_{1}+L_{o x_{2}}+\frac{d_{1} \lambda_{3} \lambda_{5}}{d_{2} d_{3} \lambda_{1}} L_{o x_{2}}-k_{3}}, L_{o x_{2}}, \frac{\lambda_{3}}{\lambda_{4}} \frac{L_{o x_{2}}+\frac{d_{1} \lambda_{3} \lambda_{5}}{d_{2} d_{3} \lambda_{1}} L_{o x_{2}}-k_{3}}{k_{1}+L_{o x_{2}}+\frac{d_{1} \lambda_{3} \lambda_{5}}{d_{2} d_{3} \lambda_{1}} L_{o x_{2}}-k_{3}}, \frac{d_{1} \lambda_{3}}{d_{2} \lambda_{1}} L_{o x_{2}}, \frac{d_{1} \lambda_{3} \lambda_{5}}{d_{2} d_{3} \lambda_{1}} L_{o x_{2}}-k_{3}\right) .
$$

Proposition 4.1. When $\lambda_{2}=P_{0}=0$, the system (4.22)-(4.26) always has two fixed points, $E_{0}$ and $E_{1}$, belonging to the plane $C_{3}=0$, and two other fixed points, $E_{u}$ and $E_{2}$, in the plane $C_{3}=\frac{\lambda_{5}}{d_{3}} M_{1}-k_{3}$ provided that conditions $A, B$ and $C$ are satisfied.

Stability analysis: The stability analysis of the fixed points is investigated analytically for $E_{0}$ and $E_{1}$, and numerically for $E_{u}$ and $E_{2}$.

We start by considering the case where $\alpha>\alpha_{0}$.

At $(0,0,0,0,0)$, the Jacobian matrix reads:

$$
J=\left[\begin{array}{ccccc}
-k_{L} & \frac{\lambda_{1}}{k_{1}} & 0 & 0 & \frac{\lambda_{1}}{k_{1}} \\
k_{L} & -d_{1} & 0 & 0 & 0 \\
0 & \frac{\lambda_{3}}{k_{1}} & -\lambda_{4} & 0 & \frac{\lambda_{1}}{k_{1}} \\
0 & 0 & \lambda_{4} & -d_{2} & 0 \\
0 & 0 & 0 & 0 & -d_{3}
\end{array}\right] .
$$

Clearly, the point $(0,0,0,0,0)$ is stable if and only if $d_{1} k_{1}>\lambda_{1}$. 

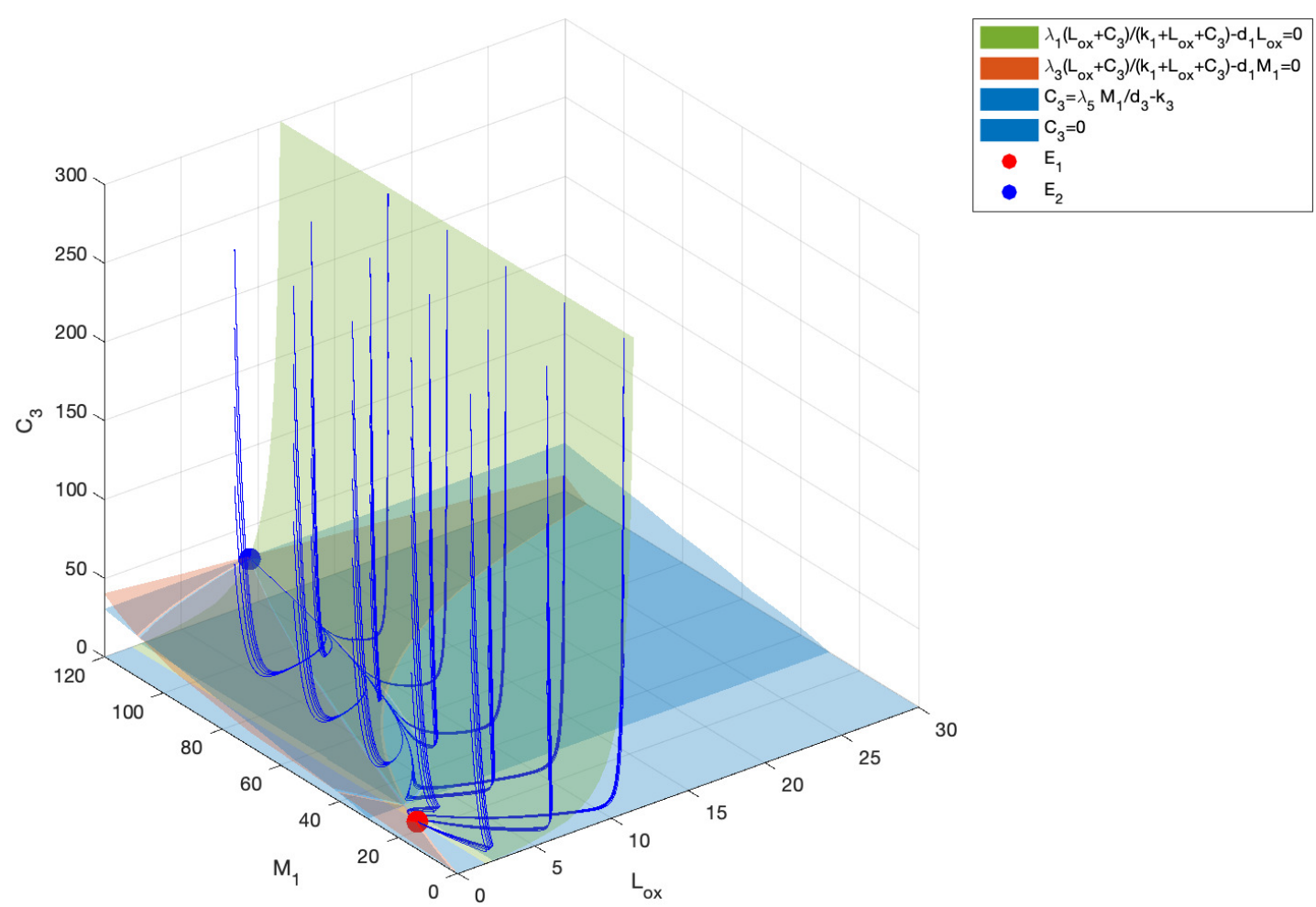

FiguRE 4. Numerical simulations showing the stability of the fixed points when conditions A, $\mathrm{B}$ and $\mathrm{C}$ are satisfied and $\lambda_{1}<\frac{d_{1} k_{1} \lambda_{3} \lambda_{5}}{\lambda_{3} \lambda_{5}-d_{2} d_{3} k_{3}}$. The trajectories are obtained by exhibiting the system of ODE's from different starting points.

At the point $\left(\frac{\lambda_{1}}{k_{L}} \frac{\lambda_{1}-d_{1} k_{1}}{\lambda_{1}}, \frac{\lambda_{1}-d_{1} k_{1}}{d_{1}}, \frac{\lambda_{3}}{\lambda_{4}} \frac{\lambda_{1}-d_{1} k_{1}}{\lambda_{1}}, \frac{\lambda_{3}}{d_{2} \lambda_{1}}\left(\lambda_{1}-d_{1} k_{1}\right), 0\right)$, the Jacobian matrix becomes:

$$
J=\left[\begin{array}{ccccc}
-k_{L} & \frac{k_{1}}{\lambda_{1}} & 0 & 0 & \frac{k_{1}}{\lambda_{1}} \\
k_{L} & -d_{1} & 0 & 0 & 0 \\
0 & \frac{\lambda_{3} k_{1}}{\lambda_{1}^{2}} & -\lambda_{4} & 0 & \frac{\lambda_{3} k_{1}}{\lambda_{1}^{2}} \\
0 & 0 & \lambda_{4} & -d_{2} & 0 \\
0 & 0 & 0 & 0 & \frac{\lambda_{5} \lambda_{3}\left(\lambda_{1}-d_{1} k_{1}\right)}{k_{3} d_{2} \lambda_{1}}-d_{3}
\end{array}\right]
$$

Therefore, this point is stable if and only if $d_{1} k_{1}<\lambda_{1}$ and $\frac{\lambda_{5} \lambda_{3}\left(\lambda_{1}-d_{1} k_{1}\right)}{k_{3} d_{2} \lambda_{1}}<d_{3}$.

We deduce from above that $E_{0}$ is always unstable. While $E_{1}$ is stable if $d_{1} k_{1}>\lambda_{1}$ or if $d_{1} k_{1}<\lambda_{1}$ and $\frac{\lambda_{5} \lambda_{3}\left(\lambda_{1}-d_{1} k_{1}\right)}{k_{3} d_{2} \lambda_{1}}<d_{3}$. We also notice that if $d_{1} k_{1}<\lambda_{1}, \lambda_{5}>\frac{d_{2} d_{3} k_{3}}{\lambda_{3}}$ and $\frac{\lambda_{5} \lambda_{3}\left(\lambda_{1}-d_{1} k_{1}\right)}{k_{3} d_{2} \lambda_{1}}<d_{3}$ then the point $E_{1}$ is stable in the plane $C_{3}=0$. Regarding the stability of the points $E_{u}$ and $E_{2}$, the numerical simulations show that $E_{u}$ is always unstable and $E_{2}$ is always stable when they exist. Figure 4 shows the numerical simulations and highlights the stability of the fixed points.

When $\alpha<\alpha_{0}$, clearly this system has only one stable equilibrium $(0,0,0,0,0)$. 
TABLE 2. Stability of the fixed points for $\alpha>\alpha_{0}$.

\begin{tabular}{|c|c|c|c|c|c|c|}
\hline & \multirow{2}{*}{\multicolumn{2}{|c|}{$\begin{array}{c}\text { Conditions A, B } \\
\text { and } \mathrm{C} \text { are all satisfied } \\
\lambda_{5}>\frac{d_{2} d_{3} k_{3}}{\lambda_{3}}\end{array}$}} & \multicolumn{4}{|c|}{$\begin{array}{l}\text { Conditions } \mathrm{A}, \mathrm{B} \\
\text { and } \mathrm{C} \text { are not all satisfied }\end{array}$} \\
\hline & & & \multicolumn{2}{|c|}{$\lambda_{5}<\frac{d_{2} d_{3} k_{3}}{\lambda_{3}}$} & \multicolumn{2}{|c|}{$\lambda_{5}>\frac{d_{2} d_{3} k_{3}}{\lambda_{3}}$} \\
\hline & $\lambda_{1}<\frac{d_{1} k_{1} \lambda_{3} \lambda_{5}}{\lambda_{3} \lambda_{5}-d_{2} d_{3} k_{3}}$ & $\lambda_{1}>\frac{d_{1} k_{1} \lambda_{3} \lambda_{5}}{\lambda_{3} \lambda_{5}-d_{2} d_{3} k_{3}}$ & $\lambda_{1}<d_{1} k_{1}$ & $\lambda_{1}>d_{1} k_{1}$ & $\lambda_{1}<\frac{d_{1} k_{1} \lambda_{3} \lambda_{5}}{\lambda_{3} \lambda_{5}-d_{2} d_{3} k_{3}}$ & $\lambda_{1}>\frac{d_{1} k_{1} \lambda_{3} \lambda_{5}}{\lambda_{3} \lambda_{5}-d_{2} d_{3} k_{3}}$ \\
\hline$E_{1}$ & stable & $\begin{array}{c}\text { stable } \\
\text { in } C_{3}=0\end{array}$ & stable & stable & stable & $\begin{array}{c}\text { stable } \\
\text { in } C_{3}=0\end{array}$ \\
\hline$E_{u}$ & unstable & unstable & $\nexists$ & $\nexists$ & $\nexists$ & $\nexists$ \\
\hline$E_{2}$ & stable & stable & $\nexists$ & $\nexists$ & $\nexists$ & $\nexists$ \\
\hline
\end{tabular}

Table 2 shows a classification of the fixed points existence and stability when $\alpha>\alpha_{0}$, according to the values of parameters.

We readily notice that when conditions $\mathrm{A}, \mathrm{B}$ and $\mathrm{C}$ are satisfied, then $\lambda_{5}>\frac{d_{2} d_{3} k_{3}}{\lambda_{3}}$ since otherwise:

$$
\begin{aligned}
& \frac{d_{1} \lambda_{3} \lambda_{5}}{d_{2} d_{3} \lambda_{1}} L_{o x_{2}}-k_{3}< \frac{d_{1} k_{3}}{\lambda_{1}} L_{o x_{2}}-k_{3} \\
&= \frac{k_{3}}{2 \lambda_{1}\left(1+\frac{d_{1} \lambda_{3} \lambda_{5}}{d_{2} d_{3} \lambda_{1}}\right)}\left(-\lambda_{1}\left(1+\frac{d_{1} \lambda_{3} \lambda_{5}}{d_{2} d_{3} \lambda_{1}}\right)-d_{1}\left(k_{1}-k_{3}\right)\right. \\
&\left.+\sqrt{\left(\lambda_{1}\left(1+\frac{d_{1} \lambda_{3} \lambda_{5}}{d_{2} d_{3} \lambda_{1}}\right)-d_{1}\left(k_{1}-k_{3}\right)\right)^{2}-4 d_{1} k_{3} \lambda_{1}\left(1+\frac{d_{1} \lambda_{3} \lambda_{5}}{d_{2} d_{3} \lambda_{1}}\right)}\right) \\
&<0 .
\end{aligned}
$$

Biological interpretations: The fixed points $E_{1}, E_{u}$ and $E_{2}$ have the following biological interpretations:

- $E_{1}$ belongs to the plane $C_{3}=0$. It corresponds the state where no pro-inflammatory cytokines are elaborated. Since the key role of modulating inflammation is attributed to cytokines, then this point conforms to the disease free situation. If $\alpha<\alpha_{0}$, this fixed point is always stable while if $\alpha>\alpha_{0}$, it is stable when $\lambda_{1}<\frac{d_{1} k_{1} \lambda_{3} \lambda_{5}}{\lambda_{3} \lambda_{5}-d_{2} d_{3} k_{3}}$ or when $\lambda_{1}>d_{1} k_{1}$ and $\lambda_{3} \lambda_{5}\left(\lambda_{1}-d_{1} k_{1}\right)<d_{2} d_{3} \lambda_{1} k_{3}$.

- $E_{2}$ belongs to the plane $C_{3}=\frac{\lambda_{5} M_{1}}{d_{3}}-k_{3}$. It represents the case where LDL, ox-LDL, inflammatory immune cells and cytokines arise in the lesion. They identify the development of the inflammatory processes. Thus the point $E_{2}$ corresponds to the inflammatory state. When $\alpha>\alpha_{0}, E_{2}$ is always stable when it exists.

- $E_{u}$ belongs to the plane $C_{3}=\frac{\lambda_{5} M 1}{d_{3}}-k_{3}$. It represents a threshold between $E_{1}$ and $E_{2}$ and is always unstable.

The conditions shown in Table 2 are interpreted as follows:

- Conditions A, B and C, when satisfied, indicate that the value of the parameter $k_{1}$ is small. This can be interpreted by inadequate effectiveness of endothelial regulators role, associated with an impaired vasodilation of the endothelium with a high permeability.

- When $\lambda_{1}<d_{1} k_{1}$ or when $d_{1} k_{1}<\lambda_{1}<\frac{d_{1} k_{1} \lambda_{3} \lambda_{5}}{\lambda_{3} \lambda_{5}-d_{2} d_{3} k_{3}}$, then the rate of LDL subendothelial penetration $\left(\lambda_{1}\right)$ is moderate. Otherwise, it is considerable and critical.

- The condition $\lambda_{5}>\frac{d_{1} d_{2} k_{3}}{\lambda_{3}}$ determines a high secretion of pro-inflammatory cytokines. 
The following conclusions can be drawn from the stability analysis:

- The trancytosis of LDL across the endothelium when regulated by a healthy glycocalyx $\left(\alpha<\alpha_{0}\right)$ does not lead to subendothelial accumulation and aggregation of LDL. This prevents inflammation and avoids plaque buildup.

- However, in the presence of certain cardiovascular risks, endothelial disruption is associated with compromised glycocalyx $\left(\alpha>\alpha_{0}\right)$. The entrance of LDL in the arterial intima is then determined by endothelial vesicles, and open endothelial junctions. Therefore, LDL particles become more likely to aggregate and be retained in situ. At this stage, the inflammation may be triggered depending on the intimal LDL particles concentration and endothelial hyperpermeability.

- In case of high endothelial permeability (conditions A, B and C are satisfied), we can elaborate the following:

- Destabilization of the endothelium always requires a high release of pro-inflammatory cytokines $\left(\lambda_{5}>\right.$ $\left.\frac{d_{1} d_{2} k_{3}}{\lambda_{3}}\right)$.

- For a low rate of LDL penetration $\left(\lambda_{1}\right)$, no inflammation is triggered normally ( $E_{1}$ stable). However, endothelial hyperpermeability is a marker of vascular inflammatory state that induces the overexpression of inflammatory mediators. This may activates the inflammatory cascades within the intima: LDL oxidation, recruitment of inflammatory monocytes, macrophage accumulation, which induces LDL oxidation, and macrophage generation of inflammatory mediators. A positive feedback loop is then formed, which generates and promotes expansion of the atherosclerotic process ( $E_{2}$ stable). Therefore, even an insignificant lipid deposition may initiate the inflammation. Thus, this case highlights the fact that LDL can be involved in the development of atherosclerosis as an initiator, without necessarily being a major factor.

- A high penetration of LDL to the intima leads to inflammation $\left(E_{2}\right.$ stable). However, in patients with cytokine deficiency diseases $\left(C_{3}=0\right)$, such as asthma, dermatitis and meningitis, autoinflammatory disorders are known to occur. These disorders are characterized by unprovoked episodes of inflammation and relative lack of autoimmune pathology. Then the inflammation is not fully established ( $E_{1}$ stable). Therefore, destabilized endothelium resulting in an increased vascular permeability promotes the disease severity according to lipid deposition.

- When conditions A, B and C are not all satisfied, the endothelial permeability is considered to be moderate. In this case, the rate of LDL penetration and the rate pro-inflammatory cytokines production can acquire large or low values. We note that an excessive LDL penetration leads absolutely to a high LDL oxidation. Therefore, a large production of pro-inflammatory cytokines and/or a large penetration of LDL promote the endothelial permeability. However, due to different factors, such as nitric oxide and HDL, the endothelial permeability gets regulated. For this reason we may have a balanced endothelial permeability while the endothelial dysfunction is detected $\left(\alpha>\alpha_{0}\right)$ and the secretion of inflammatory cytokines or the penetration of LDL is high. Hence we conclude the following:

- The vascular function regulators, such as NO and HDL, exhibit vasoprotective effects and contribute in improving endothelial function and homeostasis. The improvement/reversal of endothelial dysfunction is beneficial in the inhibition of inflammatory responses.

Therefore, regulated permeability of the endothelium prevents the inflammation.

- In general, a low LDL penetration to the intima does not initiate an inflammation whereas an increased LDL penetration favors atherogenesis.

Risk zones diagram: We used data reported in the literature to display reliable results and to provide predictive outcomes. In Table 3, we list values for some parameters used in our model. Using data of Table 3 , we distinguish, in Figure 5, three different zones, according to the values of the parameters $\lambda_{1}$ and $k_{1}$. We note that the values in Table 3 verify the condition: $\lambda_{5}>\frac{d_{2} d_{3} k_{3}}{\lambda_{3}}$. 
TABLE 3. Values of some parameters.

\begin{tabular}{ccc}
\hline Parameters & Value & Source \\
\hline$\lambda_{3}$ & $1.296 \mathrm{pg} \mathrm{cm}^{-3}$ day $^{-1}$ & {$[26,42]$} \\
$\lambda_{4}$ & $8.64 \times 10^{-2}$ day $^{-1}$ & {$[42]$} \\
$\lambda_{5}$ & 4.2 day $^{-1}$ & {$[17]$} \\
$k_{3}$ & $70 \mathrm{pg} \mathrm{cm}^{-3}$ & {$[26]$} \\
$d_{1}$ & 2.0736 day $^{-1}$ & {$[42]$} \\
$d_{2}$ & $1.5 \times 10^{-2}$ day $^{-1}$ & {$[26]$} \\
$d_{3}$ & 1.188 day $^{-1}$ & {$[26]$} \\
\hline
\end{tabular}

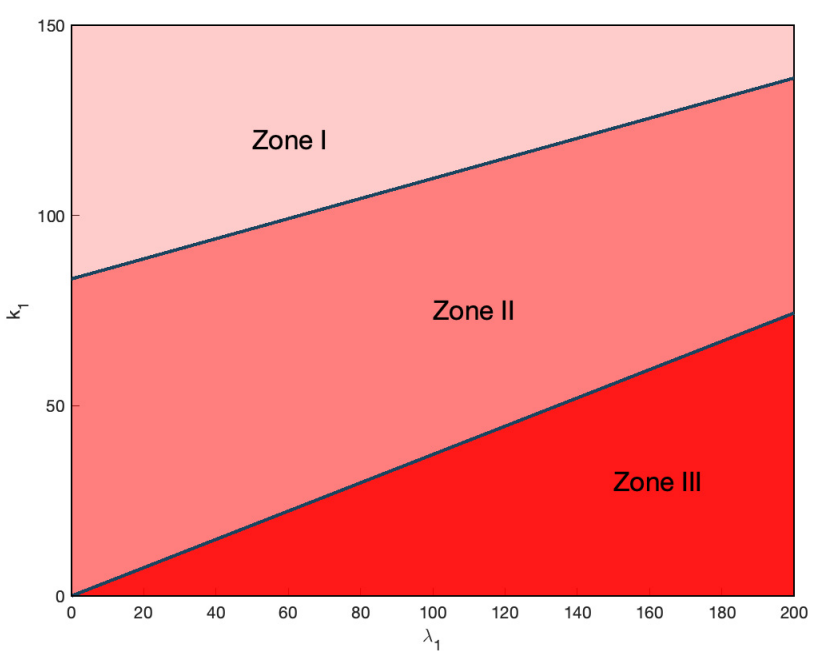

FIgURE 5. This figure shows three different zones, according to the values of the parameters $\lambda_{1}$ and $k_{1}$. Zone III is of high risk of inflammation. Zone I is a zone of low risk where the inflammation is unlikely to develop. Zone II is of intermediate risk where the inflammation may possibly occur.

- In Zone I, conditions A, B and C are not all verified and $\lambda_{1}<\frac{d_{1} k_{1} \lambda_{3} \lambda_{5}}{\lambda_{3} \lambda_{5}-d_{2} d_{3} k_{3}}$. Hence, it is a zone of low risk of inflammation.

- In Zone II, conditions A, B and C are satisfied but $\lambda_{1}<\frac{d_{1} k_{1} \lambda_{3} \lambda_{5}}{\lambda_{3} \lambda_{5}-d_{2} d_{3} k_{3}}$. It corresponds to an intermediate risk zone, when the inflammation may or may not develop.

- In Zone III, the condition $\lambda_{1}>\frac{d_{1} k_{1} \lambda_{3} \lambda_{5}}{\lambda_{3} \lambda_{5}-d_{2} d_{3} k_{3}}$ is satisfied along with conditions A, B and C. It is a zone of high risk of cardiovascular inflammation.

Thus, in this section, we study the reduced monotone system (4.27)-(4.31) for $\lambda_{2}=P_{0}=0$, where the endothelial permeability indicator is variable. The study leads to the existence of at most four fixed points $E_{0}, E_{1}, E_{u}$ and $E_{2}$. The stability analysis of the fixed points is investigated analytically for $E_{0}$ and $E_{1}$, and numerically for $E_{u}$ and $E_{2}$. It is determined by the value of $H(\alpha)$ that refers to the endothelial functionality. $E_{0}$ is always unstable. If $\alpha<\alpha_{0}$ (normal endothelium), $E_{1}$ is stable and $E_{u}$ and $E_{2}$ do not exist. For $\alpha>\alpha_{0}$, the stability analysis is summarized in Table 2 . Then we give a biological description for the equilibria, and we deduce a biological interpretation. Finally, we use data found in literature (in Tab. 3) to elaborate a risk map in Figure 5 that shows different zones of different risk levels. 


\subsubsection{Reduced model with constant $P$}

In this section we consider the term $P$ to be a $P=H(\alpha) p$ where $H(\alpha)$ is defined above and $p$ is a constant. The kinetic system of system (4.15)-(4.19) is then equivalent to the following system:

$$
\begin{gathered}
M_{1}=f_{1}\left(L_{o x}\right):=\frac{d_{1} L_{o x}+\left(\lambda_{3}-\lambda_{1}\right) P}{d_{2}}, \\
M_{1}=f_{2}\left(L_{o x}\right):=\frac{\lambda_{3} P\left(k_{2}+L_{o x}\right)}{\left(\lambda_{2}+d_{2}\right) L_{o x}+d_{2} k_{2}}, \\
\lambda_{5} \frac{C_{3}}{k_{3}+C_{3}} M_{1}-d_{3} C_{3}=0, \\
L=\frac{\lambda_{1}}{k_{L}} P, \\
A_{1}=\frac{\lambda_{3}}{\lambda_{4}} P .
\end{gathered}
$$

The intersection points between $f_{1}$ and $f_{2}$ satisfy the following equation in $L_{o x}$ :

$$
d_{1}\left(\lambda_{2}+d_{2}\right) L_{o x}^{2}+\left(\lambda_{2} \lambda_{3} P+d_{1} d_{2} k_{2}-\lambda_{1}\left(\lambda_{2}+d_{2}\right) P\right) L_{o x}-\lambda_{1} d_{2} k_{2} P=0
$$

Equation (4.44) admits a positive discriminant. Therefore, it has two real solutions, having a negative product. The positive solution is:

$$
L_{o x_{e}}=\frac{-\left(\lambda_{2} \lambda_{3} P+d_{1} d_{2} k_{2}-\lambda_{1}\left(\lambda_{2}+d_{2}\right) P\right)+\sqrt{\left(\lambda_{2} \lambda_{3} P+d_{1} d_{2} k_{2}-\lambda_{1}\left(\lambda_{2}+d_{2}\right) P\right)^{2}+4 d_{1} \lambda_{1} d_{2} k_{2}\left(\lambda_{2}+d_{2}\right) P}}{2 d_{1}\left(\lambda_{2}+d_{2}\right)} .
$$

Hence, the fixed points of system (4.15)-(4.19), for $P=H(\alpha) p$, are: $E_{1}=\left(\frac{\lambda_{1}}{k_{L}} P, L_{o x_{e}}, \frac{\lambda_{3}}{\lambda_{4}} P, M_{1_{e}}, 0\right)$ and $E_{2}=$ $\left(\frac{\lambda_{1}}{k_{L}} P, L_{o x_{e}}, \frac{\lambda_{3}}{\lambda_{4}} P, M_{1_{e}}, \frac{\lambda_{5} M_{1_{e}}}{d_{3}}-k_{3}\right)$, where $M_{1_{e}}=f_{1}\left(L_{o x_{e}}\right)=f_{2}\left(L_{o x_{e}}\right)>0$. The Jacobian matrix of system (4.15)-(4.19) reads as follows:

$$
J=\left[\begin{array}{ccccc}
-k_{L} & 0 & 0 & 0 & 0 \\
k_{L} & -\lambda_{2} \frac{k_{2} M_{1}}{\left(k_{2}+L_{o x}\right)^{2}}-d_{1} & 0 & -\lambda_{2} \frac{L_{o x}}{k_{2}+L_{o x}} & 0 \\
0 & 0 & -\lambda_{4} & 0 & 0 \\
0 & -\lambda_{2} \frac{k_{2} M_{1}}{\left(k_{2}+L_{o x}\right)^{2}} & \lambda_{4} & -\lambda_{2} \frac{L_{o x}}{k_{2}+L_{o x}}-d_{2} & 0 \\
0 & 0 & 0 & \lambda_{5} \frac{C_{3}}{k_{3}+C_{3}} & \lambda_{5} \frac{k_{3} M_{1}}{\left(k_{3}+C_{3}\right)^{2}}-d_{3}
\end{array}\right] .
$$


The eigenvalues $\eta_{i}^{\prime}$ s of $E_{1}$ are solutions of the following equation in $\eta$ :

$$
\begin{gathered}
\left(\lambda_{5} \frac{M_{1_{e}}}{k_{3}}-d_{3}-\eta\right)\left(-k_{L}-\eta\right)\left(-\lambda_{4}-\eta\right) . \\
{\left[\left(-\lambda_{2} \frac{L_{o x_{e}}}{k_{2}+L_{o x_{e}}}-d_{2}-\eta\right)\left(-\frac{\lambda_{2} k_{2} M_{1_{e}}}{\left(k_{2}+L_{o x_{e}}\right)^{2}}-d_{1}-\eta\right)-\frac{\lambda_{2}^{2} k_{2} L_{o x_{e}} M_{1_{e}}}{\left(k_{2}+L_{o x_{e}}\right)^{3}}\right]=0}
\end{gathered}
$$

Let us examine the equation:

$$
\left(-\lambda_{2} \frac{L_{o x_{e}}}{k_{2}+L_{o x_{e}}}-d_{2}-\eta\right)\left(-\frac{\lambda_{2} k_{2} M_{1_{e}}}{\left(k_{2}+L_{o x_{e}}\right)^{2}}-d_{1}-\eta\right)-\frac{\lambda_{2}^{2} k_{2} L_{o x_{e}} M_{1_{e}}}{\left(k_{2}+L_{o x_{e}}\right)^{3}}=0
$$

If we denote $a=\lambda_{2} \frac{L_{o x_{e}}}{k_{2}+L_{o x_{e}}}$ and $b=\frac{\lambda_{2} k_{2} M_{1_{e}}}{\left(k_{2}+L_{o x_{e}}\right)^{2}}$, the latter equation can be written as follows:

$$
\eta^{2}+\eta\left(a+d_{2}+b+d_{1}\right)+a d_{1}+b d_{2}+d_{1} d_{2}=0 .
$$

Its corresponding discriminant is: $D=\left(a+d_{2}-\left(b+d_{1}\right)\right)^{2}+4 a b>0$. Then there are two real solutions having a positive product and a negative sum. Therefore, this equation admits two real negative solutions.

We deduce that the point $E_{1}$ is stable if $\lambda_{5} \frac{M_{1_{e}}}{d_{3}}<k_{3}$.

Similarly, the point $E_{2}$ is stable if $\frac{d_{3}^{2} k_{3}}{\lambda_{5} M_{1_{e}}}<d_{3}$, which is equivalent to $\frac{\lambda_{5} M_{1_{e}}}{d_{3}}>k_{3}$.

Since we are considering only positive solutions, then the point $E_{2}$ is stable when it exists. When $E_{2}$ does not exist, then $E_{1}$ becomes stable.

Proposition 4.2. If $\alpha<\alpha_{0}$, the only fixed point is $E_{1}=(0,0,0,0,0)$ and it is stable. While if $\alpha>\alpha_{0}$, there may be either one fixed point $E_{1}$ or two fixed points $E_{1}$ and $E_{2} . E_{2}$ exists when $\frac{\lambda_{5} M_{1_{e}}}{d_{3}}-k_{3}>0$ and is stable when it exists. Whereas $E_{1}$ always exists and is unstable when $E_{2}$ exists.

We notice that:

$$
\begin{aligned}
\frac{\lambda_{5} M_{1_{e}}}{d_{3}}-k_{3}>0 & \Longleftrightarrow \frac{\lambda_{5}\left(d_{1} L_{o x_{e}}+\left(\lambda_{3}-\lambda_{1}\right) P\right)}{d_{3} d_{2}}-k_{3}>0 \\
& \Longleftrightarrow \lambda_{5}\left(d_{1} L_{o x_{e}}+\lambda_{3} P\right)>d_{3} d_{2} k_{3}+\lambda_{5} \lambda_{1} P
\end{aligned}
$$

Then the inequality $\frac{\lambda_{5} M_{1_{e}}}{d_{3}}>k_{3}$ correlates with a high penetration of LDL into the intima (high $\lambda_{1}$ ).

As shown in the section above, $E_{1}$ and $E_{2}$ illustrate the disease free and the inflammatory states respectively. The following conclusions derive from this study:

- When no endothelial dysfunction is detected, the inflammation does not develop ( $E_{1}$ stable).

- Otherwise, if the penetration of LDL to the intima is moderate, then no inflammation is triggered $\left(E_{1}\right.$ stable). But, a high penetration of LDL leads to the inflammation $\left(E_{2}\right.$ stable).

We recall that the above results are found for $\lambda_{2} \geq 0$ and $P_{0} \geq 0$. This reduced model with a constant permeability indicator term shows that we are able to retrieve the results of Section 4.1.1 even for strictly positive values of $\lambda_{2}$ and $P_{0}$.

As a conclusion for this section, we study the reduced system (4.27)-(4.31) for $\lambda_{2}>0$ and $P_{0}>0$ where the endothelial permeability indicator is constant. The analysis leads to the existence of at most two fixed points 
$E_{1}$ and $E_{2}$. The stability analysis of the fixed points is investigated analytically and is determined by the value of $H(\alpha)$ that refers to the endothelial functionality. If $\alpha<\alpha_{0}$ (normal endothelium), $E_{1}$ is the only fixed point and it stable. Otherwise, $E_{1}$ and $E_{2}$ are the fixed points. When $E_{1}$ is stable, $E_{2}$ is unstable, and vice versa. Then we give a biological description for the equilibria, and we deduce a biological interpretation of the results. The conclusions drawn from this model are in line with the results of the previous subsection.

\section{Perturbed solutions}

In this section, we investigate the existence and the stability of solutions for system (4.22)-(4.26) when $P_{0}>0$ and $\lambda_{2}>0$ by applying the implicit function theorem.

We start by fixing $\lambda_{2}=0$ and considering $P_{0}>0$ in equations (4.22)-(4.26). First, we check the existence of a solution near $E_{1}$ when $P_{0}$ is in a small neighborhood of 0 then we investigate its stability. We retrieve the same results for $E_{2}$ by proceeding in the same manner.

Let the operator $A\left(P_{0}, U\right): B(0,1) \times C^{2}([0,1]) \rightarrow C^{0}([0,1])$ be given in a neighborhood $D$ of the point $\left(0, E_{1}\right) \in B(0,1) \times C^{2}([0,1])$ such that $A\left(P_{0}, U\right)=F(U)+P_{0} f(x)$, where:

$$
U=\left[\begin{array}{c}
L \\
L_{o x} \\
A_{1} \\
M_{1} \\
C_{3}
\end{array}\right], F(U)=\left[\begin{array}{c}
\lambda_{1} H(\alpha) \frac{L_{o x}+C_{3}}{k_{1}+L_{o x}+C_{3}}-k_{L} L \\
k_{L} L-d_{1} L_{o x} \\
\lambda_{3} H(\alpha) \frac{L_{o x}+C_{3}}{k_{1}+L_{o x}+C_{3}}-\lambda_{4} A_{1} \\
\lambda_{4} A_{1}-d_{2} M_{1} \\
\lambda_{5} \frac{C_{3}}{k_{3}+C_{3}} M_{1}-d_{3} C_{3}
\end{array}\right] \text { and } f(x)=\left[\begin{array}{c}
\lambda_{1} H(\alpha) \frac{1}{k_{1}+L_{o x}+C_{3}} \\
0 \\
\lambda_{3} H(\alpha) \frac{1}{k_{1}+L_{o x}+C_{3}} \\
0 \\
0
\end{array}\right]
$$

We assume that $U(x)$ is a non negative-valued function of class $C^{2}([0,1])$ such that $U(0)=0$ and $U(1)=0$. The function $F(U)$ is of class $C^{1}$ and $F\left(E_{1}\right)=0$.

We have that $A\left(0, E_{1}\right)=0$. Moreover, the eigenvalues of the Jacobian matrix of system (4.22)-

(4.26) at $E_{1}$, are $-d_{2},-\lambda_{4}$,

$$
\frac{-k_{L}-d_{1}+\sqrt{\left(k_{L}-d_{1}\right)^{2}+4 k_{L} \frac{\lambda_{1}}{k_{1}}}}{2}, \frac{-k_{L}-d_{1}+\sqrt{\left(k_{L}-d_{1}\right)^{2}+4 k_{L} \frac{\lambda_{1}}{k_{1}}}}{2} \text { and }
$$

$\frac{d_{1} \lambda_{3} \lambda_{5}}{d_{2} \lambda_{1} k_{3}} \frac{\lambda_{1}-d_{1} k_{1}+\sqrt{\left(\lambda_{1}-d_{1} k_{1}\right)^{2}}}{2 d_{1}}-d_{3}$, and none of these eigenvalues is zero.

Then there exists an operator $\Phi$ given in some neighborhood $G \subset B(0,1)$ of the point 0 such that it maps this neighborhood into the space $C^{2}([0,1])$ and satisfies the following properties:

1. $A\left(P_{0}, \Phi\left(P_{0}\right)\right)=0$ in $G$.

2. $\Phi(0)=E_{1}$.

3. $\Phi$ is continuous at 0 .

Let us denote $F_{1}=\Phi\left(E_{1}\right)$. Particularly, when $P_{0} \in G$ and $P_{0}>0$, the system admits a solution $F_{1}$ near $E_{1}$. We note that, even when $E_{1}=(0,0,0,0,0), F_{1}$ lies in the positive half-space. In fact, when $P_{0}>0$, in the plane $C_{3}=0$, the fixed points $E_{0}$ and $E_{1}$ of the system are the intersection points between $M_{1}=\frac{d_{1} \lambda_{3}}{d_{2} \lambda_{1}} L_{o x}$ and $M_{1}=\frac{\lambda_{3}}{d_{2}} \frac{P_{0}+L_{o x}}{k_{1}+L_{o x}}$. Therefore, the $L_{o x}$-coordinate of $E_{0}$ and $E_{1}$ are $\frac{\lambda_{1}-d_{1} k_{1}-\sqrt{\left(\lambda_{1}-d_{1} k_{1}\right)^{2}+4 \lambda_{1} P_{0} d_{1}}}{2 d_{1}}$ and $\frac{\lambda_{1}-d_{1} k_{1}+\sqrt{\left(\lambda_{1}-d_{1} k_{1}\right)^{2}+4 \lambda_{1} P_{0} d_{1}}}{2 d_{1}}$ respectively. Clearly, the $L_{o x}$-coordinate of $E_{1}$ is strictly positive.

In order to check the stability of $F_{1}$, we consider the Jacobian matrix of the kinetic system (4.22)-(4.26) for $\lambda_{2}=0$ at $F_{1}$. It is a square matrix whose elements depend smoothly on $P_{0}$. By Theorem A.3 of the appendix, if 
$\lambda_{0}$ is a simple eigenvalue for $P_{0}=0$ at $E_{1}$, then for all $\tau$ near 0 there is a corresponding eigenvalue that depends smoothly on $P_{0}$. Thus, since the eigenvalues at $E_{1}$ are all simple, when $P_{0}$ is small enough, each eigenvalue of the Jacobian matrix at $F_{1}$ lies in a small neighborhood which center is the corresponding eigenvalue of the Jacobian matrix at $E_{1}$. In other words, the eigenvalues of the Jacobian at $F_{1}$ are all real, simple and have the same sign as the eigenvalues at $E_{1}$. Then, we can deduce that $F_{1}$ is stable when $E_{1}$ is stable and when the parameter $P_{0}$ is close enough to 0 .

Similarly, in order to confirm that the system (4.22)-(4.26) has two fixed points $G_{1}$ and $G_{2}$ when $P_{0}>0$ and $\lambda_{2}>0$, we consider the operator $A^{*}\left(\lambda_{2}, U\right): B(0,1) \times C^{2}([0,1]) \rightarrow C^{0}([0,1])$ be given in a neighborhood $\mathcal{D}$ of the point $\left(0, F_{1}\right) \in B(0,1) \times C^{2}([0,1])$ such that $A^{*}\left(P_{0}, U\right)=F(U)+\lambda_{2} f^{*}(x)$, where:

$$
U=\left[\begin{array}{c}
L \\
L_{o x} \\
A_{1} \\
M_{1} \\
C_{3}
\end{array}\right], F(U)=\left[\begin{array}{c}
\lambda_{1} H(\alpha) \frac{P_{0}+L_{o x}+C_{3}}{k_{1}+L_{o x}+C_{3}}-k_{L} L \\
k_{L} L-d_{1} L_{o x} \\
\lambda_{3} H(\alpha) \frac{P_{0}+L_{o x}+C_{3}}{k_{1}+L_{o x}+C_{3}}-\lambda_{4} A_{1} \\
\lambda_{4} A_{1}-d_{2} M_{1} \\
\lambda_{5} \frac{C_{3}}{k_{3}+C_{3}} M_{1}-d_{3} C_{3}
\end{array}\right] \text { and } f^{*}(x)=\left[\begin{array}{c}
0 \\
\frac{L_{o x}}{k_{2}+L_{o x}} M_{1} \\
0 \\
\frac{L_{o x}}{k_{2}+L_{o x}} M_{1} \\
0
\end{array}\right]
$$

We proceed in the same manner as above, and we can even conclude that $G_{1}$ and $G_{2}$ are stable respectively, when $E_{1}$ and $E_{2}$ are stable.

Hence, this section is devoted to the application of the Implicit Function Theorem to the reduced model (4.22)-(4.26). We start by considering the reduced model for $\lambda_{2}=0$ and $P_{0}$ sufficiently small. We prove, using the Implicit Function Theorem, the existence of two fixed points $F_{1}$ and $F_{2}$ near $E_{1}$ and $E_{2}$ respectively. Then we show that the stability analysis for $F_{1}$ and $F_{2}$ correlates with the stability analysis for $E_{1}$ and $E_{2}$, by applying the Implicit Function Theorem one more time. Then, for $\lambda_{2}$ and $P_{0}$ being sufficiently small, we proceed similarly, to prove the existence and stability of the two fixed points $G_{1}$ and $G_{2}$ near $F_{1}$ and $F_{2}$.

\section{TRAVELING WAVE SOLUTIONS}

In this section, we study the existence of traveling wave solutions of the reaction-diffusion system (4.22)-(4.26) for $\lambda_{2}=P_{0}=0$. The system becomes:

$$
\begin{gathered}
\frac{\partial L}{\partial t}=D_{1} \frac{\partial^{2} L}{\partial x^{2}}+\lambda_{1} H(\alpha) \frac{L_{o x}+C_{3}}{k_{1}+L_{o x}+C_{3}}-k_{L} L, \\
\frac{\partial L_{o x}}{\partial t}=D_{3} \frac{\partial^{2} L_{o x}}{\partial x^{2}}+k_{L} L-d_{1} L_{o x}, \\
\frac{\partial A_{1}}{\partial t}=D_{4} \frac{\partial^{2} A_{1}}{\partial x^{2}}+\lambda_{3} H(\alpha) \frac{L_{o x}+C_{3}}{k_{1}+L_{o x}+C_{3}}-\lambda_{4} A_{1}, \\
\frac{\partial M_{1}}{\partial t}=D_{6} \frac{\partial^{2} M_{1}}{\partial x^{2}}+\lambda_{4} A_{1}-d_{2} M_{1},
\end{gathered}
$$




$$
\frac{\partial C_{3}}{\partial t}=D_{12} \frac{\partial^{2} C_{3}}{\partial x^{2}}+\lambda_{5} \frac{C_{3}}{k_{3}+C_{3}} M_{1}-d_{3} C_{3}
$$

Consider the following problem:

$$
\frac{\partial U}{\partial t}=d \frac{\partial^{2} U}{\partial x^{2}}+F(U)
$$

where $U=\left(U_{1}, \ldots, U_{m}\right), F=\left(F_{1}, \ldots, F_{m}\right)$ and $d$ is a diagonal matrix with positive diagonal elements.

A traveling wave solution of this latter system is a particular solution which has the form

$$
u(x, t):=\omega(x-c t)
$$

where the constant $c$ is the speed of the wave. We assume that the traveling wave solution has the following limits:

$$
\lim _{x \rightarrow \pm \infty} \omega(x)=\omega_{ \pm}
$$

Traveling waves usually describe transition processes from one fixed point to another. The existence of traveling wave solutions of reaction-diffusion systems is ensured in either monostable or bistable cases if the system is monotone, i.e. the off-diagonal elements of its Jacobian are positive (see [56], Chap. 4, Thms. 1.6 and 1.7). These results can be extended if the off-diagonal elements of the Jacobian are non negative.

The following theorem gives the existence of waves in the bistable case.

Theorem 6.1. Suppose that $F\left(\omega_{+}\right)=F\left(\omega_{-}\right)=0$, where $\omega_{+}<\omega_{-}$(the inequality is component-wise) and the matrices $F^{\prime}\left(\omega_{ \pm}\right)$have all eigenvalues in the left half- plane. Suppose also that there exists a finite number of points $\omega^{j} \neq \omega_{ \pm}, j=1, \cdots, k$ such that $\omega_{+} \leq \omega^{j} \leq \omega_{-}, F^{\prime}\left(\omega^{j}\right)=0$ and each matrix $F^{\prime}\left(\omega^{j}\right)$ has at least one eigenvalue in the right half-plane. If the matrices $F^{\prime}\left(\omega^{j}\right)$ are irreducible with their principal eigenvalues found in the right half-plane then there exist a constant $c$ and a unique monotonically decreasing traveling wave solution $u(x, t)=\omega(x-c t)$ of system (6.6) with the limits $\omega( \pm \infty)=\omega_{ \pm}$.

The following theorem gives the existence of waves in the monostable case.

Theorem 6.2. Suppose that $F\left(\omega_{+}\right)=F\left(\omega_{-}\right)=0$, where $\omega_{+}<\omega_{-}$(the inequality is component-wise), and there are no other zeros of the function $F(\omega)$ for $\omega_{+} \leq \omega \leq \omega_{-}$. Assume that the matrix $F^{\prime}\left(\omega_{-}\right)$has all eigenvalues in the left half-plane while the matrix $F^{\prime}\left(\omega_{+}\right)$has an eigenvalue with a positive real part. Then there exists a constant $c^{*}$ such that $\forall c \in\left[c^{*}, \infty[\right.$, there exists a monotonically decreasing traveling wave solution $u(x, t)=\omega(x-c t)$ of system (6.6) of velocity $c$ with the limits $\omega( \pm \infty)=\omega_{ \pm}$.

Proof. For a detailed proof of these theorems, one can refer to [56], Chapter 4, Theorem 1.6 and Theorem 1.7.

In what follows, we establish the existence of traveling wave solutions of system (6.1)-(6.5), then we show some numerical results that illustrate the wave propagation. 
In order to study traveling wave solutions of system (6.1)-(6.5), we take:

$$
U=\left[\begin{array}{c}
L \\
L_{o x} \\
A_{1} \\
M_{1} \\
C_{3}
\end{array}\right], F(U)=\left[\begin{array}{c}
\lambda_{1} H(\alpha) \frac{L_{o x}+C_{3}}{k_{1}+L_{o x}+C_{3}}-k_{L} L \\
k_{L} L-d_{1} L_{o x} \\
\lambda_{3} H(\alpha) \frac{L_{o x}+C_{3}}{k_{1}+L_{o x}+C_{3}}-\lambda_{4} A_{1} \\
\lambda_{4} A_{1}-d_{2} M_{1} \\
\lambda_{5} \frac{C_{3}}{k_{3}+C_{3}} M_{1}-d_{3} C_{3}
\end{array}\right] \text { and } d=\left[\begin{array}{ccccc}
D_{1} & 0 & 0 & 0 & 0 \\
0 & D_{3} & 0 & 0 & 0 \\
0 & 0 & D_{4} & 0 & 0 \\
0 & 0 & 0 & D_{6} & 0 \\
0 & 0 & 0 & 0 & D_{12}
\end{array}\right] .
$$

A traveling wave solution is of the form:

$$
\left(\begin{array}{c}
L(x, t) \\
L_{o x}(x, t) \\
A_{1}(x, t) \\
M_{1}(x, t) \\
C_{3}(x, t)
\end{array}\right):=\omega(x-c t)
$$

where the constant $c$ is the speed of the wave. In our study, such solutions describe propagation of the inflammation.

We are interested in bounded solutions of system (6.1)-(6.5) on the whole axis. We consider the theoretical space domain to be the entire real line $]-\infty, \infty[$ and we assume that the traveling wave solution have limits at infinity:

$$
\lim _{x \rightarrow \pm \infty} \omega(x)=\omega_{ \pm}
$$

with $\omega_{+}$and $\omega_{-}$being two fixed points of system $(6.1)-(6.5)$.

Proposition 6.3. The vector-valued function $F(U)$ satisfies the condition $\frac{\partial F_{i}}{\partial U_{j}} \geq 0, i, j=1, \ldots, 5, i \neq j$, where $U_{j}$ is the $j^{\text {th }}$ component of $U$.

Proof. The Jacobian matrix of system (6.1)-(6.5) reads:

$$
J=\left[\begin{array}{ccccc}
-k_{L} & \lambda_{1} H(\alpha) \frac{k_{1}}{\left(k_{1}+L_{o x}+C_{3}\right)^{2}} & 0 & 0 & \lambda_{1} H(\alpha) \frac{k_{1}}{\left(k_{1}+L_{o x}+C_{3}\right)^{2}} \\
k_{L} & \lambda_{1} H(\alpha) \frac{k_{1}}{\left(k_{1}+L_{o x}+C_{3}\right)^{2}} & -\lambda_{4} & 0 & \lambda_{3} H(\alpha) \frac{k_{1}}{\left(k_{1}+L_{o x}+C_{3}\right)^{2}} \\
0 & 0 & \lambda_{4} & -d_{2} & 0 \\
0 & 0 & 0 & \lambda_{5} \frac{C_{3}}{k_{3}+C_{3}} & \lambda_{5} \frac{k_{3} M_{1}}{\left(k_{3}+C_{3}\right)^{2}}-d_{3}
\end{array}\right]
$$

The off-diagonal elements of $J$ are non negative.

Theorem 6.4. Suppose that conditions $A, B$ and $C$ are satisfied. Suppose also that $\lambda_{5}>\frac{d_{2} d_{3} k_{3}}{\lambda_{3}}$ and $\lambda_{1}<$ $\frac{d_{1} k_{1} \lambda_{3} \lambda_{5}}{\lambda_{3} \lambda_{5}-d_{2} d_{3} k_{3}}$. If $\lambda_{1} \neq d_{1}\left(k_{1}+k_{3}-\frac{\lambda_{3} \lambda_{5}}{d_{2} d_{3}}+2 \sqrt{k_{3} k_{1}}\right)$ and $\lambda_{1} \neq d_{1}\left(k_{1}+k_{3}-\frac{\lambda_{3} \lambda_{5}}{d_{2} d_{3}}-2 \sqrt{k_{3} k_{1}}\right)$ then there exist a constant $c$ and a unique monotonically decreasing traveling wave solution $u(x, t)=\omega(x-c t)$ of system (6.1)(6.5) with the limits $\omega( \pm \infty)=\omega_{ \pm}$where $\omega_{+}$and $\omega_{-}$are the stable fixed points of the system. 


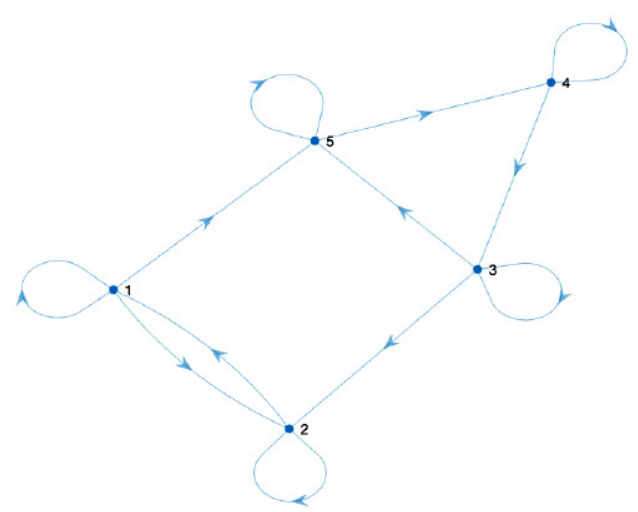

FiguRE 6. Directed graph of the Jacobian matrix at $E_{u}$, in the bistable case.

Proof. Suppose that conditions A, B and C are verified and that $\lambda_{5}>\frac{d_{2} d_{3} k_{3}}{\lambda_{3}}$ and $\lambda_{1}<\frac{d_{1} k_{1} \lambda_{3} \lambda_{5}}{\lambda_{3} \lambda_{5}-d_{2} d_{3} k_{3}}$, then the latter problem admits two different stable fixed points $E_{1}$ and $E_{2}$ and one unstable fixed point $E_{u}$. If we denote by $\omega_{+}=E_{1}, \omega_{-}=E_{2}$, and $\omega_{1}=E_{u}$, then $F\left(\omega_{+}\right)=F\left(\omega_{-}\right)=0, \omega_{+}<\omega_{-}$(the inequality is component-wise) and the matrices $F^{\prime}\left(\omega_{ \pm}\right)$have all eigenvalues in the left half- plane. Moreover, there exists one point $\omega^{1} \neq \omega_{ \pm}$, such that $\omega_{+} \leq \omega^{1} \leq \omega_{-}, F\left(\omega^{1}\right)=0$ and the matrix $F^{\prime}\left(\omega^{1}\right)$ has at least one eigenvalue in the right half-plane. In addition to that, $F^{\prime}\left(E_{u}\right)$ is irreductible since it has a strongly connected digraph as shown in Figure 6 . Since $E_{u}$ is unstable then the corresponding Jacobian has at least one eigenvalue of non negative real part. Therefore, its principal eigenvalue has a nonnegative real part. To ensure that this eigenvalue lies in the right half-space, we impose that the derterminant of the Jacobian matrix at $E_{u}$ is different than 0 . This latter condition is equivalent to having $\lambda_{1} \neq d_{1}\left(k_{1}+k_{3}-\frac{\lambda_{3} \lambda_{5}}{d_{2} d_{3}}+2 \sqrt{k_{3} k_{1}}\right)$ and $\lambda_{1} \neq d_{1}\left(k_{1}+k_{3}-\frac{\lambda_{3} \lambda_{5}}{d_{2} d_{3}}-2 \sqrt{k_{3} k_{1}}\right)$. Therefore, by Theorem 6.1 there exists, in the bistable case, a constant $c$ and a monotonically decreasing traveling wave solution $u(x, t)=\omega_{(x-c t)}$ of system (6.1)-(6.5) with the limits $\omega( \pm \infty)=\omega_{ \pm}$if $\lambda_{1} \neq d_{1}\left(k_{1}+k_{3}-\frac{\lambda_{3} \lambda_{5}}{d_{2} d_{3}}+2 \sqrt{k_{3} k_{1}}\right)$ and $\lambda_{1} \neq d_{1}\left(k_{1}+k_{3}-\frac{\lambda_{3} \lambda_{5}}{d_{2} d_{3}}-2 \sqrt{k_{3} k_{1}}\right)$.

We note that if $\lambda_{1}=d_{1}\left(k_{1}+k_{3}-\frac{\lambda_{3} \lambda_{5}}{d_{2} d_{3}}+2 \sqrt{k_{3} k_{1}}\right)$ or $\lambda_{1}=d_{1}\left(k_{1}+k_{3}-\frac{\lambda_{3} \lambda_{5}}{d_{2} d_{3}}-2 \sqrt{k_{3} k_{1}}\right)$, the reduced system admits a traveling wave solution in the bistable case, if the Jacobian matrix at $E_{u}$ has at least an eigenvalue of strictly positive real part.

Theorem 6.5. If conditions $A, B$ and $C$ are satisfied, $\lambda_{5}>\frac{d_{2} d_{3} k_{3}}{\lambda_{3}}$ and $\lambda_{1}>\frac{d_{1} k_{1} \lambda_{3} \lambda_{5}}{\lambda_{3} \lambda_{5}-d_{2} d_{3} k_{3}}$ or if not all conditions $A, B$ and $C$ are satisfied, then there exists a constant $c^{*}$ such that $\forall c \in\left[c^{*}, \infty[\right.$, there exists a monotonically decreasing traveling wave solution $u(x, t)=\omega(x-c t)$ of system (6.1)-(6.5) of velocity $c$ with the limits $\omega( \pm \infty)=$ $\omega_{ \pm}$, where $\omega_{+}$and $\omega_{-}$are the fixed points of the system.

Proof. Under the conditions of the latter theorem, system (6.1)-(6.5) admits one stable fixed point $E_{1}$ and one unstable fixed point $E_{2}$. We let $\omega_{+}=E_{2}, \omega_{-}=E_{1}$. Then, we have $F\left(\omega_{+}\right)=F\left(\omega_{-}\right)=0, \omega_{+}<\omega_{-}$(the inequality is component-wise), and there are no other zeros of the function $F(\omega)$ for $\omega_{+} \leq \omega \leq \omega_{-}$. Moreover, $F^{\prime}\left(\omega_{-}\right)$has all eigenvalues in the left half-plane while the matrix $F^{\prime}\left(\omega_{+}\right)$has an eigenvalue with a positive real part. Then, by Theorem 6.2 , there exists a constant $c^{*}$ such that $\forall c \in\left[c^{*}, \infty[\right.$, there exists a monotonically decreasing traveling wave solution $u(x, t)=\omega(x-c t)$ of system (6.1)-(6.5) of velocity $c$ with the limits $\omega( \pm \infty)=\omega_{ \pm}$. 


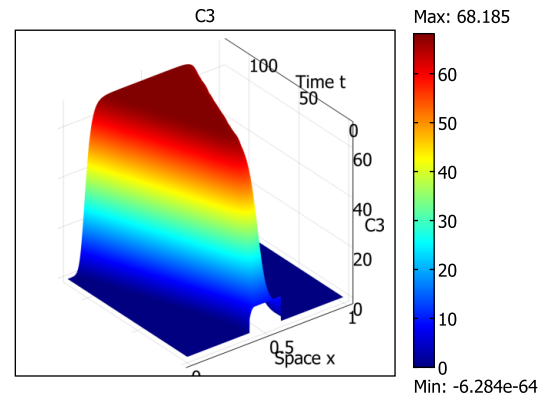

(A)

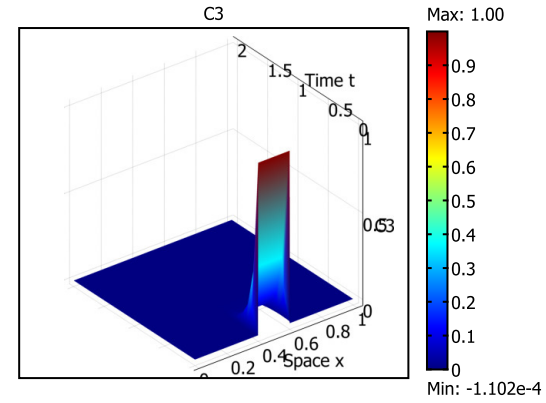

(B)

FIGURE 7. Numerical simulations showing the wave propagation of $C_{3}$ when conditions A, B and $\mathrm{C}$ are satisfied and $\lambda_{1}<\frac{d_{1} k_{1} \lambda_{3} \lambda_{5}}{\lambda_{3} \lambda_{5}-d_{2} d_{3} k_{3}}$. A large perturbation of the non inflammatory state leads to the propagation of an inflammatory reaction and a small perturbation of the non inflammatory state does not lead to the propagation of an inflammatory reaction.

The numerical solution of system (6.1)-(6.5) on the 1D interval ]0, 1[ with homogeneous Neumann boundary conditions for the bistable case is shown in Figure 7.

In conclusion, in this section, we prove the existence of traveling wave solution for the monotone reduced system (6.1)-(6.5) in both bistable case and monostable. Theorem 6.4 proves the existence of a traveling wave solution for system (6.1)-(6.5) in the bistable case and Theorem 6.5 proves the existence of a traveling wave solution for system (6.1)-(6.5) in the monostable case.

\section{Perturbed Waves in the Bistable CASE}

A traveling wave solution of speed $c$ for problem

$$
\frac{\partial U}{\partial t}=a \frac{\partial^{2} U}{\partial x^{2}}+F(U),
$$

where $a$ is a constant matrix, that is of the form $U(x, t):=\omega(x-c t)$, is a solution for

$$
a \omega^{\prime \prime}+c \omega^{\prime}+F(\omega)=0
$$

Suppose that $F$ is continuous together with its third derivatives. Suppose that for some $c=c_{0}$, there exists a solution $w_{0}(x)$ of this system with limits at infinity

$$
\lim _{x \rightarrow \pm \infty} \omega_{0}(x)=\omega_{ \pm}
$$

We will study persistence of solutions under small perturbations of the system. We consider the operator

$$
\mathcal{A}(\omega, c)=a \omega^{\prime \prime}+c \omega+F(\omega)
$$

acting from $C^{2+D}(\mathbb{R}) \times \mathbb{R}$ into $C^{D}(\mathbb{R}), 0<D<1$, and the linearized operator

$$
L u=a u^{\prime \prime}+c u^{\prime}+F^{\prime}\left(\omega_{0}(x)\right) u .
$$


The essential spectrum is the set of complex numbers $\lambda$ satisfying the algebraic equation

$$
\operatorname{det}\left(-a \xi^{2}+c i \xi+F^{\prime}\left(\omega_{ \pm}\right)-\lambda E\right)=0, \xi \in \mathbb{R}
$$

Here $\mathrm{E}$ is the identity matrix.

Theorem 7.1. Suppose that problem (7.2)-(7.3) has a solution $\omega_{0}(x)$ for some $c=c_{0}$. If the zero eigenvalue of the linearized operator $L$ given by (7.5) is simple and equation (7.6) with respect to $\xi$ does not have solutions for any real number $\lambda \geq 0$, then for all $\epsilon$ sufficiently small the problem

$$
a \omega^{\prime \prime}+c \omega^{\prime}+G_{\epsilon}(\omega)=0, \omega( \pm \infty)=\omega_{ \pm}^{\epsilon}
$$

where $G_{\epsilon}(\omega)=F(\omega)+\epsilon g(\omega)$ and $G_{\epsilon}\left(\omega_{ \pm}^{\epsilon}\right)=0$, has a solution $\omega_{\epsilon}$ for some $c=c_{\epsilon}$. Here $\omega_{ \pm}^{\epsilon} \rightarrow \omega_{ \pm}, c_{\epsilon} \rightarrow c_{0}$ and $\left\|\omega_{\epsilon}-\omega_{0}\right\|_{C^{2+D}(\mathbb{R})}$ as $\epsilon \rightarrow 0$. The functions $F$ and $g$ are uniformly bounded and continuous together with their third derivatives.

Proof. For a detailed proof of the theorem, one can refer to [56], Chapter 4, Theorem 1.23.

System (6.1)-(6.5) for $D_{1}=D_{3}=D_{4}=D_{6}=D_{12}=a$ can be written

$$
\frac{\partial U}{\partial t}=a \frac{\partial^{2} U}{\partial x^{2}}+F(U)
$$

where

$$
U=\left[\begin{array}{c}
L \\
L_{o x} \\
A_{1} \\
M_{1} \\
C_{3}
\end{array}\right], \text { and } F(U)=\left[\begin{array}{c}
\lambda_{1} H(\alpha) \frac{L_{o x}+C_{3}}{k_{1}+L_{o x}+C_{3}}-k_{L} L \\
k_{L} L-d_{1} L_{o x} \\
\lambda_{3} H(\alpha) \frac{L_{o x}+C_{3}}{k_{1}+L_{o x}+C_{3}}-\lambda_{4} A_{1} \\
\lambda_{4} A_{1}-d_{2} M_{1} \\
\lambda_{5} \frac{C_{3}}{k_{3}+C_{3}} M_{1}-d_{3} C_{3}
\end{array}\right]
$$

The function $F(U)$ is continuous together with its third derivative. A traveling wave solution of velocity $c$ of this problem is of the form $\omega(x-c t)=U(x, t)$. We consider as well

$$
f(U)=\left[\begin{array}{c}
\lambda_{1} H(\alpha) \frac{1}{k_{1}+L_{o x}+C_{3}} \\
0 \\
\lambda_{3} H(\alpha) \frac{1}{k_{1}+L_{o x}+C_{3}} \\
0 \\
0
\end{array}\right] \text { and } f^{*}(U)=\left[\begin{array}{c}
0 \\
\frac{L_{o x}}{k_{2}+L_{o x}} M_{1} \\
0 \\
\frac{L_{o x}}{k_{2}+L_{o x}} M_{1} \\
0
\end{array}\right] .
$$

When conditions A, B and C are satisfied, $\lambda_{5}>\frac{d_{2} d_{3} k_{3}}{\lambda_{3}}$ and $\lambda_{1}<\frac{d_{1} k_{1} \lambda_{3} \lambda_{5}}{\lambda_{3} \lambda_{5}-d_{2} d_{3} k_{3}}$, system (6.1)-(6.5) admits two stable equilibria $E_{1}$ and $E_{2}$ and a unique traveling wave solution $\omega_{0}$ of velocity $c=c_{0}$ connecting $E_{1}$ to $E_{2}$. The solution $\omega_{0}$ verifies equation $(7.2)$ and $\lim _{x \rightarrow \pm \infty} \omega_{0}(x)=\omega_{ \pm}$where $\omega_{+}=E_{1}$ and $\omega_{-}=E_{2}$. 
Theorem 7.2. When conditions $A, B$ and $C$ are satisfied, $\lambda_{5}>\frac{d_{2} d_{3} k_{3}}{\lambda_{3}}$ and $\lambda_{1}<\frac{d_{1} k_{1} \lambda_{3} \lambda_{5}}{\lambda_{3} \lambda_{5}-d_{2} d_{3} k_{3}}$, then for all $P_{0}$ sufficiently small the problem

$$
a \omega^{\prime \prime}+c \omega^{\prime}+G_{P_{0}}(\omega)=0, \omega( \pm \infty)=\omega_{ \pm}^{P_{0}},
$$

where $G_{P_{0}}(\omega)=F(\omega)+P_{0} f(\omega), G_{P_{0}}\left(\omega_{ \pm}^{P_{0}}\right)=0$, has a solution $\omega_{P_{0}}$ for some $c=c_{P_{0}}$. Here $\omega_{ \pm}^{P_{0}} \rightarrow \omega_{ \pm}$where $\omega_{+}=E_{1}$ and $\omega_{-}=E_{2}, c_{P_{0}} \rightarrow c_{0}$ and $\left\|\omega_{P_{0}}-\omega_{0}\right\|_{C^{2+D}(\mathbb{R})} \rightarrow 0$ as $P_{0} \rightarrow 0$. The functions $F$ and $g$ are uniformly bounded and continuous together with their third derivatives.

Proof. The linearized operator $L u=a u^{\prime \prime}+c u^{\prime}+F^{\prime}\left(\omega_{0}(x)\right) u$ has a zero eigenvalue of eigenfunction the derivative of $\omega_{0}$, the unique traveling wave solution for system (6.1)-(6.5) under the conditions of the theorem. This eigenvalue is simple since it has a one-dimensional eigenspace. The essential spectrum given by

$$
\operatorname{det}\left(-a \xi^{2}+c i \xi+F^{\prime}\left(E_{1}\right)-\lambda E\right)=0, \xi \in \mathbb{R} .
$$

is the set of $\lambda_{i}=\eta_{i}-a \xi^{2}+c i \xi$, where $\eta_{i}$ is an eigenvalue of the Jacobian matrix of system (6.1)-(6.5) at $E_{1}$, for $i=1, \cdots, 5$. The eigenvalues $\eta_{i}$ 's are all real negative since the point $E_{1}$ is stable. Therefore, the essential spectrum lies in the left half-plane and for any real number $\lambda \geq 0$, equation (7.10) with respect to $\xi$ does not have solutions. Similarly, the essential spectrum given by $\operatorname{det}\left(-a \xi^{2}+c i \xi+F^{\prime}\left(E_{2}\right)-\lambda E\right)=0, \xi \in \mathbb{R}$ lies in the left half-plane. Thus, by Theorem 7.1, for all $P_{0}$ sufficiently small, problem (7.7) has a solution $\omega_{P_{0}}$ for some $c=c_{P_{0}}$. Here $\omega_{ \pm}^{P_{0}} \rightarrow \omega_{ \pm}$where $\omega_{+}^{P_{0}}=F_{1}, \omega_{-}^{P_{0}}=F_{2}, \omega_{+}=E_{1}$ and $\omega_{-}=E_{2}, c_{P_{0}} \rightarrow c_{0}$ and $\left\|\omega_{P_{0}}-\omega_{0}\right\|_{C^{2+D}(\mathbb{R})}$ as $P_{0} \rightarrow 0$. The functions $F$ and $g$ are uniformly bounded and continuous together with their third derivatives.

Theorem 7.3. When conditions $A, B$ and $C$ are verified, $\lambda_{5}>\frac{d_{2} d_{3} k_{3}}{\lambda_{3}}$ and $\lambda_{1}<\frac{d_{1} k_{1} \lambda_{3} \lambda_{5}}{\lambda_{3} \lambda_{5}-d_{2} d_{3} k_{3}}$, then for all $\lambda_{2}$ sufficiently small the problem

$$
a \omega^{\prime \prime}+c \omega^{\prime}+G_{\lambda_{2}}(\omega)=0, \omega( \pm \infty)=\omega_{ \pm}^{\lambda_{2}},
$$

where $G_{\lambda_{2}}(\omega)=G_{P_{0}}(\omega)+\lambda_{2} f^{*}(\omega), G_{\lambda_{2}}\left(\omega_{ \pm}^{\lambda_{2}}\right)=0$, has a solution $\omega_{\lambda_{2}}$ for some $c=c_{\lambda_{2}}$. Here $\omega_{ \pm}^{\lambda_{2}} \rightarrow \omega_{ \pm}^{P_{0}}$, $c_{\lambda_{2}} \rightarrow c_{P_{0}}$ and $\left\|\omega_{\lambda_{2}}-\omega_{P_{0}}\right\|_{C^{2+D}(\mathbb{R})} \rightarrow 0$ as $\lambda_{2} \rightarrow 0$. The functions $G_{P_{0}}$ and $f^{*}$ are uniformly bounded and continuous together with their third derivatives.

Proof. The linearized operator $L u=a u^{\prime \prime}+c u^{\prime}+G_{P_{0}}^{\prime}\left(\omega_{P_{0}}(x)\right) u$ has a zero eigenvalue of eigenfunction the derivative of $\omega_{P_{0}}$, the unique traveling wave solution for problem (7.7) under the conditions of the theorem. This eigenvalue is simple since it has a one-dimensional eigenspace. The essential spectrum, given by

$$
\operatorname{det}\left(-a \xi^{2}+c i \xi+G_{P_{0}}^{\prime}\left(F_{1}\right)-\zeta E\right)=0, \xi \in \mathbb{R},
$$

is the set of $\zeta_{i}=\mu_{i}-a \xi^{2}+c i \xi$, where $\mu_{i}$ is an eigenvalue of the Jacobian matrix of problem (7.7) at $F_{1}$, for $i=1, \cdots, 5$. The eigenvalues $\mu_{i}$ 's are all real negative since the point $F_{1}$ is stable. Therefore, the essential spectrum lies in the left half-plane and for any real $\zeta \geq 0$, equation (7.10) with respect to $\xi$ does not have solutions. Similarly, the essential spectrum given by $\operatorname{det}\left(-a \xi^{2}+c i \xi+G_{P_{0}}^{\prime}\left(F_{2}\right)-\zeta E\right)=0, \xi \in \mathbb{R}$ lies in the left half-plane.

Thus, by Theorem 7.1, for all $\lambda_{2}$ sufficiently small, problem (7.9) has a solution $\omega_{\lambda_{2}}$ for some $c=c_{\lambda_{2}}$. Here $\omega_{ \pm}^{\lambda_{2}} \rightarrow \omega_{ \pm}^{P_{0}}$ where $\omega_{+}^{\lambda_{2}}=G_{1}, \omega_{-}^{\lambda_{2}}=G_{2}, \omega_{+}^{P_{0}}=F_{1}$ and $\omega_{-}^{P_{0}}=F_{2}, c_{\lambda_{2}} \rightarrow c_{P_{0}}$ and $\left\|\omega_{\lambda_{2}}-\omega_{P_{0}}\right\|_{C^{2+D}(\mathbb{R})}$ as $\lambda_{2} \rightarrow 0$. The functions $G_{P_{0}}$ and $f^{*}$ are uniformly bounded and continuous together with their third derivatives.

To summarize, in this section, we prove the existence of perturbed traveling wave solution for system (4.22)(4.26) in the bistable case. Theorem 7.2 proves the existence of a traveling wave solution for system (4.22)-(4.26) 
when $\lambda_{2}=0$ and $P_{0}$ is sufficiently small under the conditions of the bistable case. Theorem 7.3 proves the existence of a traveling wave solution for system (4.22)-(4.26) when $\lambda_{2}$ and $P_{0}$ are sufficiently small in the bistable case.

\section{TRANSITION FROM THE REDUCED MODEL TO THE COMPLETE MODEL}

In this section, we first examine the consistency of existence and stability of fixed points analysis of the complete model (4.1)-(4.14) for some given values of the parameters with the analysis of the reduced model (4.22)-(4.26), and we analyze bifurcation diagrams for the complete model. Next, we investigate the effect of the anti-inflammatory process on the behavior of the complete system by altering the value of the parameter $\lambda_{P A_{2}}$ that generates the anti-inflammatory responses in system (4.1)-(4.14).

To begin with, the parameters of the complete system (4.1)-(4.14) in this section are chosen in an appropriate way to ensure the existence of fixed points. For this aim we assume that the parameters $k_{H}, d_{A_{2}}, d_{M_{2}}, d_{T_{1}}, d_{T_{2}}$, $d_{C_{1}}, d_{C_{2}}, d_{C_{4}}$ and $d_{F}$ are sufficiently big. For simplicity, we assume that they are all equal to 1 . Moreover, the values of $T_{1}^{0}$ and $T_{2}^{0}$ are chosen conveniently to impose the positivity of the fixed points components $C_{1}$ and $C_{2}$.

We fix some parameters as follows: $\sigma_{H}=10^{-9}, H_{0}=10^{-9}, k_{H}=1, A_{2}^{0}=1, \lambda_{L_{o x} M_{2}}=10, k_{C_{1}}=0, k_{4}=$ $0, \lambda_{C_{2} A_{2}}=1, k_{C_{2}}=1, d_{A_{2}}=1, d_{M_{2}}=1, \lambda_{P T_{1}}=1, T_{1}^{0}=15, d_{T_{1}}=1, \lambda_{P T_{2}}=1, T_{2}^{0}=15, d_{T_{2}}=1, \lambda_{C_{1}}=1, d_{C_{1}}=$ $1, \lambda_{C_{2}}=1, d_{C_{2}}=1, k=0, \lambda_{C_{4} F}=10^{-9}, \lambda_{C_{4}}=10^{-9}, d_{C_{4}}=1, d_{F}=1, k_{C_{4}}=1$ and $\alpha>\alpha_{0}$. We vary the remaining parameters by considering the conditions of Table 2 .

The numerical study the ODE's system for the complete model shows that the analysis of fixed points existence and stability is identical to the one for the reduced model. In other words, the fixed points of the complete model verify the classification of Table 2 for $\lambda_{L_{o x} M_{1}}$ and $P_{0}$ being equal 0 or positive and close enough to 0 .

Moreover, the common components between the fixed points of the complete model and the ones of the reduced model have the same values under the same conditions of Table 2 for very small values of $\lambda_{P A_{2}}$. Additionally, the study of the PDEs system of the complete model indicates the existence of traveling wave solution having same velocity as the traveling wave solution found for the reduced model, under the same conditions of Table 2, as shown in subfigures (a) and (b) of Figure 11.

We denote by $P_{1}$ and $P_{2}$ the solutions for the complete model that correspond to $E_{1}$ and $E_{2}$ respectively, the solutions of the reduced model. $P_{1}$ corresponds to the disease free state and $P_{2}$ refers to the inflammatory state.

Therefore, for a certain range of values of some parameters, there is rigorous conformity in the stability analysis between the complete model and the reduced one. By assigning small values to the parameter $\lambda_{P A_{2}}$, a significant similarity in the components values of fixed points of the complete and reduced systems is perceived. Further, we noticed that the propagation of the traveling wave solutions for the reduced and complete models are identical and have the same velocities. This highlights that, for a certain range of parameters values, the inductive approach consisting of reducing the complete model and making broad generalizations of the results is valid.

The complete model provides a wider description of atherogenesis than the reduced model. To highlight the effect of endothelial permeability and intimal LDL penetration on the development of atherosclerosis, we use the bifurcation diagrams that enable the visualization of the system behavior for more general biological results.

Figure 8 shows that for a small $k_{1}$, the only stable equilibrium is $P_{2}$; for an intermediate value of $k_{1}$, the system has two stable points $P_{1}$ and $P_{2}$; and for a high value of $k_{1}, P_{1}$ is the only stable point. Explicitly, a high endothelial permeability leads to inflammation, a regulated endothelial permeability prevents the disease, and an intermediate permeability may lead to the development of atherosclerosis up to a certain threshold.

While in Figure 9, we examine the effect of LDL penetration by varying the parameter $\lambda_{1}$. Concretely, a low LDL penetration inhibits the initiation of inflammation ( $P_{1}$ stable), an intermediate LDL penetration may trigger the inflammation up to a certain threshold (bistable case), and a high LDL penetration favors the inflammation to set up ( $P_{2}$ stable). We note that in Figures 8 and 9 , the parameter $\lambda_{P A_{2}}$ is taken to be equal to 1. 


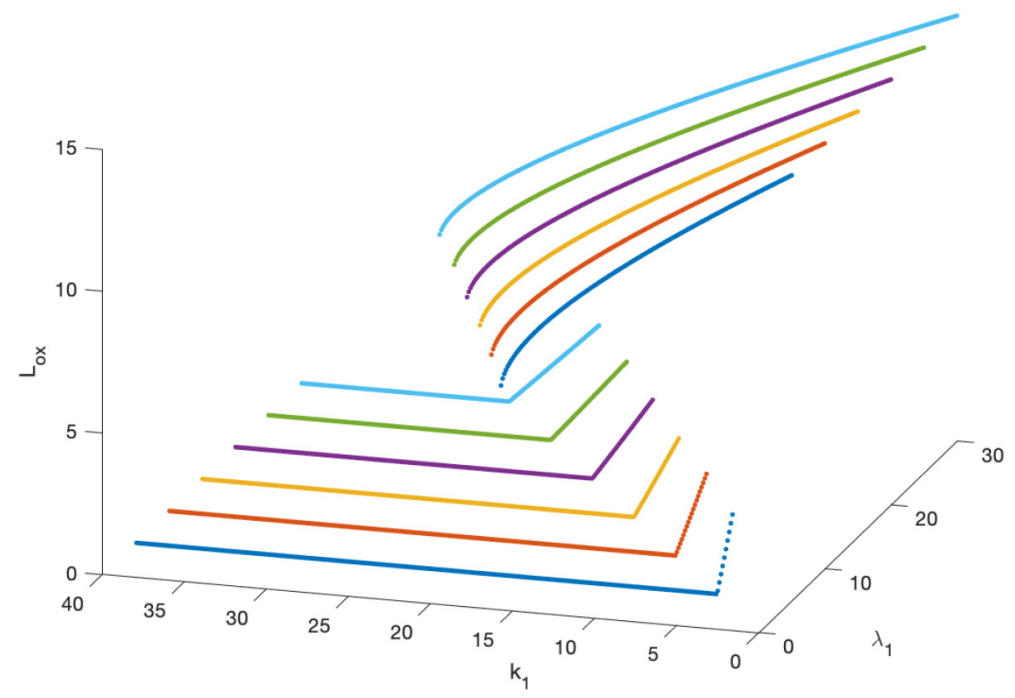

FIgURE 8. Bifurcation diagram for the complete model showing the behavior of the system when the value of the parameter $\lambda_{1}$ is fixed and the parameter $k_{1}$ is variable. The curves of same color belong to the same plane where $\lambda_{1}$ is constant.

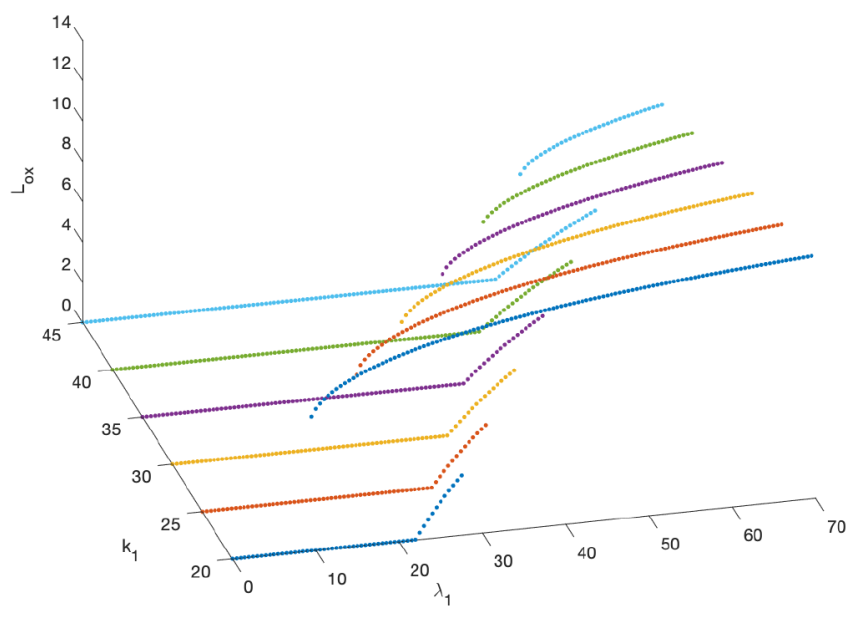

FiguRE 9. Bifurcation diagram for the complete model showing the behavior of the system when the value of the parameter $k_{1}$ is fixed and the parameter $\lambda_{1}$ is variable. The curves of same color belong to the same plane where $k_{1}$ is constant.

In order to investigate the anti-inflammatory effect on the ensue of atherogenesis, we proceed by varying the parameter $\lambda_{P A_{2}}$. Figure 10 provides the values of selected stable fixed points components, as a result of the numerical simulations of both reduced and complete models, particularly in the bistable case. Whereas, Figure 11 illustrates the projection onto the Space-Time plane for the $C_{3}$-component of the traveling wave solution for the reduced model and for the complete model with different values of $\lambda_{P A_{2}}$. The subfigures show trapezoidal form with lines that separate the blue surface from the red surface. The slopes of these lines are equal to the inverse of the velocities of the traveling wave solutions.

If we gradually expand the anti-inflammatory process by increasing the value of the parameter $\lambda_{P A_{2}}$, we identify a smooth decrease of fixed points components of pro-inflammatory mediators such as $L_{o x}, A_{1}$ and $M_{1}$ 


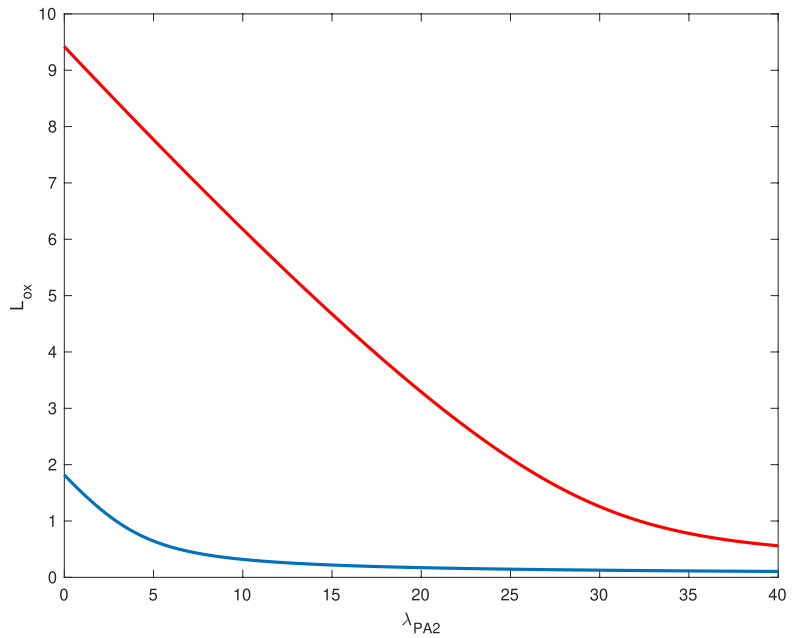

(A)

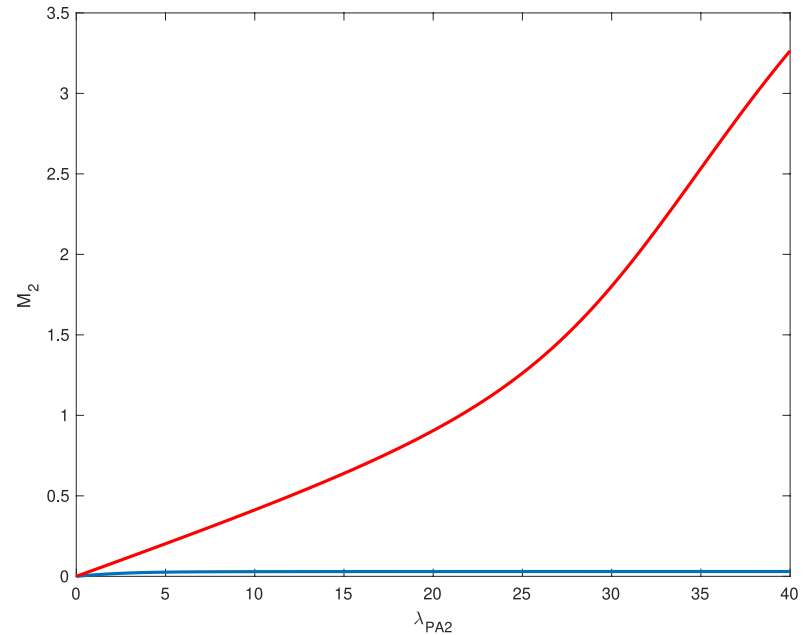

(B)

Figure 10. The curves correspond to the $L_{o x}$-coordinates of the fixed points in Figure (a) and the $M_{2}$-coordinates of the fixed points in Figure (b) as function of $\lambda_{P A_{2}}$, in the bistable case. The blue curves correspond to the coordinates of $P_{1}$ and the red curves correspond to the coordinates of $P_{2}$.

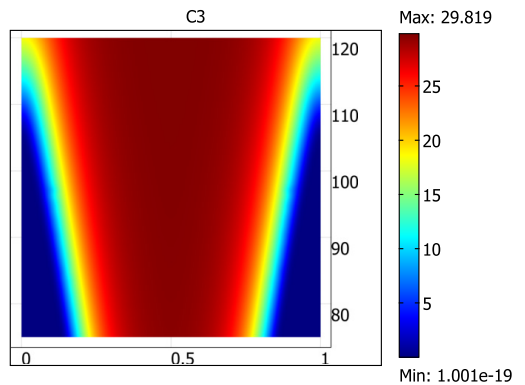

(A)

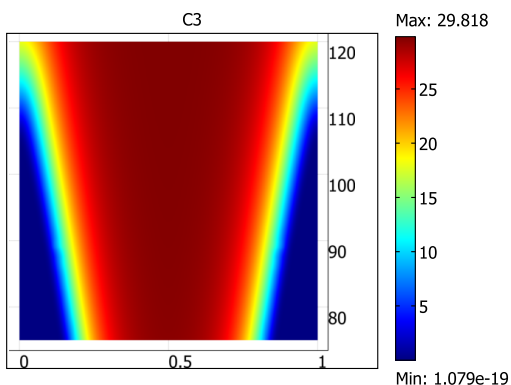

(B)

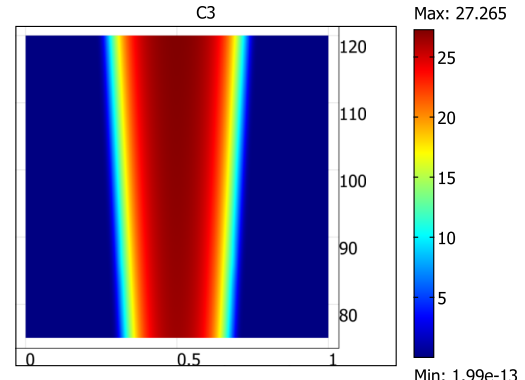

(c)

Figure 11. (a)-(c) Show the projection onto the Space-Time plane for the $C_{3}$-component of the traveling wave solution, when $\lambda_{2}=P_{0}=0$. Figure (a) corresponds to the reduced model. The other figures correspond to the complete model as follows: Figure (b) for $\lambda_{P A_{2}}=10^{-9}$ and Figure (c) for $\lambda_{P_{A_{2}}}=20$. The wave propagation projection onto the Space-Time plane shows lines that form a trapezoidal-like shape. The slopes of these lines (green edges between the blue part and the red part) are equal to the inverse of the wave velocity.

and a progressive increase of the fixed points components of anti-inflammatory mediators, such as $A_{2}$ and $M_{2}$. Likewise, the traveling wave solution propagates with a gradually decreased velocity. Biologically, this highlights the regulatory effect of the anti-inflammatory responses in atherosclerosis progression. In fact, atherosclerosis regression has a significant correlation with LDL reduction, depicted when we incorporate the anti-inflammatory effects to the model. Anti-inflammatory agents are implicated in modulating inflammatory responses and contribute to reduce inflammation progression. This explains the delay in inflammation propagation observed. Thus, in our model, the attenuation of the disease severity is attributable to the anti-inflammatory responses.

A further increase in the value of $\lambda_{P A_{2}}$ leads to a considerable decrease of the $L_{o x}$-coordinate of the fixed points and an important increase of the $M_{2}$-coordinate associated with a negative velocity of wave propagation, 


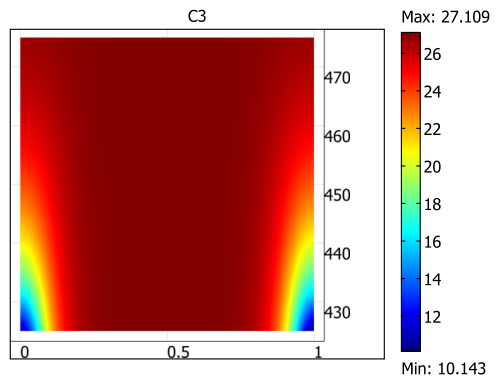

(A)

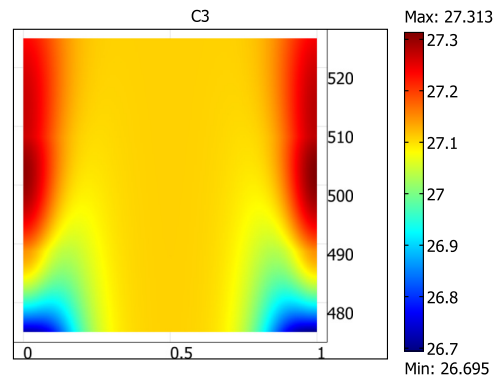

(B)

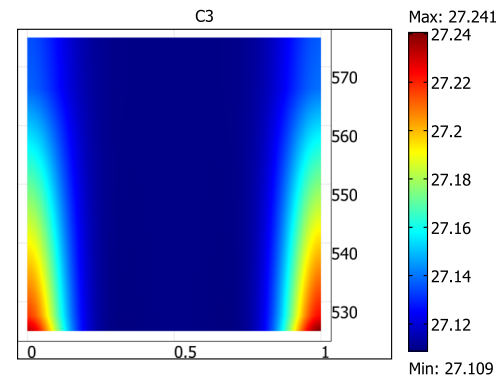

(c)

FIGURE 12. For a large value of $\lambda_{P A_{2}}\left(\lambda_{P A_{2}}>20\right)$, the velocity of the wave propagation becomes negative. Figures (a)-(c) show the wave propagation at different increasing times for a large value of $\lambda_{\mathrm{PA}_{2}}$.

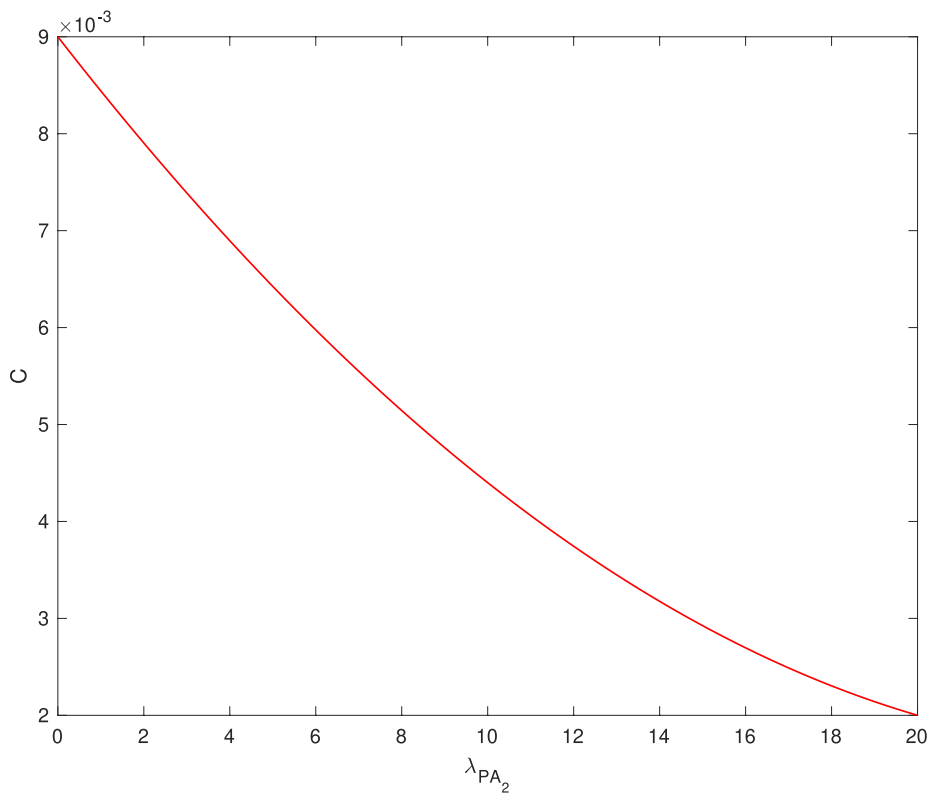

FIGURE 13. Wave velocities found for the complete model with parameters of Table 3 for different values of $\lambda_{P A_{2}}$. The space interval of the simulations corresponds to the real length of a plaque, and the velocities unit is $m m / d a y$.

as shown in Figure 12. To this magnitude of decreased wave propagation, the system behavior determines the plaque regression, that is, the reversal of the atherosclerosis process. It involves the depletion of cholesterol stores, a gradual decline in pro-inflammatory macrophage numbers and their replacement with anti-inflammatory macrophages, promoting the removal of necrotic material and tissue healing. Thus, our model emphasizes the beneficial role of anti-inflammatory effects exerted within the atherosclerotic lesion in plaque regression and stabilization.

Finally, Figure 13 shows the values of the velocities of the traveling wave solutions for the complete model with realistic parameters taken from Table 3. The simulations are exhibited by taking into account the real length of a plaque to be $8.8 \mathrm{~mm}$ as reported in [57]. This figure displays a decrease in the velocity of propagation of the inflammation when the anti-inflammatory process is amplified. We use the values of velocities in this graph and the length of a real plaque to compute the necessary time for plaque formation. We obtain the 
following results: If no anti-inflammatory responses are triggered $\left(\lambda_{P A_{2}}=0\right)$, when the endothelial dysfunction occurs, the plaque takes 2.678 years to form. However, when the anti-inflammatory processes arise, the plaque needs a notably increased number of years to establish. For instance, it takes 5.47 years to form if $\lambda_{P A_{2}}=10$ and 12.05 years if $\lambda_{P A_{2}}=20$. This result clearly confirms that an anti-inflammatory lifestyle such as exercising, quitting smoking and adopting a balanced healthy diet prevents plaque progression. Likewise, it emphasizes the role of anti-inflammatory therapeutics in treatment of atherosclerosis that proved efficient promising targets in controlling plaque development.

The results of this section are found for $\lambda_{L_{o x} M_{1}}=P_{0}=0$ and then verified for $\lambda_{L_{o x} M_{1}}=P_{0}=0.01$.

In sum, we show in this section the consistency of the existence and stability analysis of fixed points, as well as traveling wave solutions for the complete model (4.1)-(4.14) and the reduced model (4.22)-(4.26), when the parameter $\lambda_{P A_{2}}$ is sufficiently small. Figures 8 and 9 show bifurcation diagrams for the complete model and infer on the evolution of inflammation depending on the values of $\lambda_{1}$ and $k_{1}$. By increasing the value of $\lambda_{P A_{2}}$, we provide the evolution of $L_{o x}$ and $M_{2}$ coordinates of the fixed points in the bistable case in Figure 10. Figures 11 and 12 show the projection of the wave propagation onto the Space-Time plane, and give an idea about the wave propagation velocity. Finally, some conclusions on the effect of anti-inflammatory agents on inflammation regression are drawn.

\section{Discussion}

This work is devoted to the mathematical modeling of atherosclerosis. It provides an initial model that describes the anti and pro-inflammatory processes arising during the atherogenesis through partial differential equations of reaction-diffusion type. The endothelial functionality is examined and integrated to the model. A reduced model considering the pro-inflammatory process is then derived from the complete model and analyzed. It shows that the initiation of the inflammation is determined by the endothelial function and the penetration of LDL within the intima. Besides, experimental data reported from the literature contribute to providing a diagram of risk zones according to the values of two critical parameters. Likewise, numerical simulations emphasize the existence of traveling wave solutions for the reduced monotone model. The existence of perturbed solutions and perturbed traveling wave solutions are also investigated. Then the transition from reduced model to the complete initial model is studied along with graphical results. A conformity in results between the reduced and complete model is perceived for a certain range of parameters. Bifurcation diagrams show the evolution of the inflammation depending on endothelial permeability and LDL penetration to the intima. The analysis of the complete model simulations reveals a regulation of the inflammation development due to the integration of the anti-inflammatory cascades. Even though the conclusions approve and generalize previous results, this model has some limitations.

In this model many assumptions were imposed. Some of them are related to the monotonicity of the reduced model. To overcome these conditions we proved the existence of perturbed solutions. Another assumption is required to achieve the classification of Table 2 . It signifies that a high endothelial permeability is associated with a high release of pro-inflammatory cytokines. From a biological point of view, this assumption is commonly valid since endothelial dysfunction promotes endothelial activation and consequently the release of pro-inflammatory cytokines. However, due to some pathologic issues, this relationship between endothelial permeability and proinflammatory cytokines can be altered. More details on dependance of pro-inflammatory cytokines on endothelial dysfunction are beneficial to provide a more detailed classification.

The domain of this model is one-dimensional. A more accurate representation for complex conditions can be done by considering 2D and 3D models, where the artery can be represented by a rectangular or cylindrical domain, the study infers on intimal accumulation of cholesterol and plaque formation. Moreover, a fluid-structure interaction study can describe interactions between blood dynamics and the structural mechanics of the arterial wall. Since the blood's behavior is greatly affected by the deformation of the artery, the mechanical analyses are used to provide rigorous representations of flow distribution and explore possible plaque rupture mechanisms.

This model considers permeability of the endothelium through the step function $H(\alpha)$ that only takes discrete values. For a better representation of endothelial permeability, it can be distributed by a continuous function. 
When this function depends on the space $x$, the model can provide more relevant results on plaque formation. In other words, when the site of lesion is taken into account, the study may derive to conclusions on vascular sites susceptibility to atherosclerotic lesions, and consequently may confirm suggestions on regional selectivity in atherosclerotic responses to risk factors.

Clinical information is a key point to improve the efficiency of the results. Integrating reliable clinical parameters for the complete model can provide objective evidence of resulting insights. Experimental data incorporated to the model can give quantitative description of atherosclerosis and reflect realistic physiological effects suggesting the possibility of clinical use of such a model in the prediction of disease progression, decisions on endothelial recovery drug dosages, and the estimation of time of infection. Based on physiological data, this model could contribute to improving the treatment of atherosclerosis. However, obtaining accurate data for this model is a fundamental challenge.

An important biological mechanism can be included to this model: the reverse cholesterol transport (RCT). $\mathrm{RCT}$ from macrophages in atherosclerotic plaques is a critical mechanism. The excess of ox-LDL is transferred from arterial macrophages to HDL with subsequent transport to the liver for degradation. Research has provided important insights into the molecular mechanisms of RCT and showed that HDL protective function through reverse cholesterol transport likely contributes to the regression of established plaques. This model considers only the anti-inflammatory function of HDL. By considering the RCT, this model can provide more detailed results on plaque regression matched to the concentration of HDL.

Further development of atherosclerosis model might also incorporate risk factors like hypercholesterolemia, diabetes or hypertension that have a significant impact on the evolution of the plaque. Risk factors can be modeled as parameters in the mathematical model. They influence the behavior of the system, and are crucial in inducing the inflammation. By combining the risk factor effect to the model, the biological results would comprise a wider spectrum of results that confirm epidemiological, and clinical trials concerning emerging risk factors and their available therapies.

A diverse array of studies examined therapeutics for the treatment of atherosclerosis. Using statins for primary prevention is recommended by guidelines, and is prevalent to lower cardiovascular risk. Although statins were developed to specifically reduce cholesterol synthesis, clinical trials have indicated that their beneficial effects extend beyond lipid lowering. These drugs further stabilize atherosclerotic plaque. The action of statins would be included to the model that can inspect on their role in the evolution of the inflammation. This would increase the accuracy of our understanding of atherosclerosis. Such model would allow to possibly predict the efficacy of drugs and helps in optimizing the treatments.

Atherosclerotic plaques have different chemical composition as well as structures. Some of them are significantly more predisposed to rupture than others. Atherosclerotic plaque rupture is a recognized major cause of acute coronary syndrome. Such mathematical model concerns in particular the atherogenesis and the onset of plaque formation. It describes some mechanisms through which the plaque builds up. However, the characteristic components and pathogenic mechanisms of the lesions are not considered. In order to study the consequences of the plaque formation, a more descriptive model considering types of plaque and their stability would enable a more powerful mathematical approach.

As the outbreak of COVID-19 becomes the major global concern, several articles appear currently analyzing the possible correlations between COVID-19 and atherosclerosis. Possible cardiovascular manifestations of COVID-19 include acute coronary syndrome, myocardial injury, and cardiac failure. Some researches focus on the role of the pro-inflammatory state during infection and the influence of respiratory infections in atherosclerosis complications. Multiple articles are published to emphasize the link between COVID-19 and atherosclerosis . They provide a highly important knowledge on atherosclerotic plaque evolution and rupture in infected people that together with the wide range of available data afford various insights for mathematical models. An enhancement of the model of this paper would be to include the effect of COVID-19 on atherosclerotic lesions. This gives rise to a deeper understanding of the impact of COVID-19 on atherosclerotic lesions and complications and may provide a scientific framework for studies of cardiovascular manifestations of COVID-19 infection and therapeutic interventions to mitigate inflammation and improve outcomes in patients with COVID-19. 


\section{APPENDIX A.}

Theorem A.1. Let $B_{1}$ and $B_{2}$ be two Banach spaces and $(\epsilon, U) \in B_{1} \times B_{2}$. Let $x \in[0,1]$ and $U(x)$ be a function of class $C^{2}([0,1])$ such that $U(0)=0$ and $U(1)=0$. We define a function $F$ such that $F(U)$ is of class $C^{1}$ and $F(E)=0$. Let a be a constant. Suppose that the operator $A(\epsilon, U)=a U^{\prime \prime}+F(U)+\epsilon f(x)$ is given in a neighborhood $D$ of a point $(0, E) \in B_{1} \times B_{2}$, it maps it into a Banach space $F$.

If $F^{\prime}(E) \neq k^{2} \pi^{2}, \forall k \in \mathbb{N}$ then there exists an operator $\Phi$ given in some neighborhood $G \subset B_{1}$ of the point 0 such that it maps this neighborhood into the space $B_{2}$ and satisfies the following properties:

1. $A(\epsilon, \Phi(\epsilon))=0$.

2. $\Phi(0)=E$.

3. $\Phi$ is continuous at 0 .

The operator $\Phi$ is uniquely determined by these properties.

Under the conditions of the theorem, if $A$ is continuous everywhere in $D$, then the operator $\Phi$ is continuous in some neighborhood of the point 0 . If we assume, moreover, that the partial derivative $A_{\epsilon}^{\prime}$ exists in $D$ and is continuous at $(0, E)$, then the operator $\Phi$ is differentiable at 0 and

$$
\Phi^{\prime}(0)=-\left(A_{U}^{\prime}(0, E)\right)^{-1} A_{\epsilon}^{\prime}(0, E) .
$$

Proof. We have the following:

$$
A_{U}^{\prime}(\epsilon, U) V=\left[\frac{d}{\mathrm{~d} t} A(\epsilon, U+t V)\right]_{t=0}=a V^{\prime \prime}+V F^{\prime}(U)
$$

In particular,

$$
\left[\frac{d}{\mathrm{~d} t} A(\epsilon, U+t V)\right]_{U=E, t=0}=a V^{\prime \prime}+V F^{\prime}(E) .
$$

We let the operator $L: C^{2}([0,1]) \rightarrow C^{0}([0,1])$, such that $\forall V \in C^{2}([0,1]), L V=a V^{\prime \prime}+F^{\prime}(E) V$ is an approximation of $A(\epsilon, U)$ about $U=E$.

After the substitution, the boundary conditions become: $V(0)=0$ and $V(1)=0$.

We have:

- A is continuous on $D$ and particularly at $(0, E)$ and $A(0, E)=0$.

- The operator $A_{U}^{\prime}$ defined as $A_{U}^{\prime}(\epsilon, U)=a \frac{d^{2}}{d x^{2}}+F^{\prime}(U)$ exists because $F$ is of class $C^{1}$. Let us check the continuity of $A_{U}^{\prime}$ at $(0, E)$ :

To start with, $F^{\prime}$ is continuous because $F$ is of class $C^{1}$. Then there exists $D_{0}$, such that $\forall \eta>0$, if $\|U-E\|_{C^{2}}<D_{0}$, then $\left\|F^{\prime}(U)-F^{\prime}(E)\right\|<\eta$.

Let $(\epsilon, U)$ be near $(0,0)$, there exist $D_{1}$ and $D_{2}$, such that $|\epsilon|<D_{1}$ and $\|U-E\|_{C^{2}}<\min \left(D_{0}, D_{2}\right)$.

$$
\begin{aligned}
\left\|A_{U}^{\prime}(\epsilon, U)-A_{U}^{\prime}(0, E)\right\| & =\sup _{\|V\| \leq 1}\left\|\left(A_{U}^{\prime}(\epsilon, U)-A_{U}^{\prime}(0, E)\right) V\right\| \\
& =\sup _{\|V\| \leq 1}\left\|F^{\prime}(U) V-F^{\prime}(E) V\right\| \\
& \leq\left\|F^{\prime}(U)-F^{\prime}(E)\right\| \sup _{\|V\| \leq 1}\|V\|
\end{aligned}
$$




$$
\begin{aligned}
& =\left\|F^{\prime}(U)-F^{\prime}(E)\right\| \\
& \leq \eta \quad \forall \eta>0 .
\end{aligned}
$$

- $A_{U}^{\prime}(0, E)=L$ is an operator from $B_{2}$ into $F$. To find the spectrum of $L$, we let $\lambda$ be an eigenvalue of $L$, then for $V \in C^{2}([0,1]), L V=\lambda V$ gives the following:

$$
a V^{\prime \prime}+\left(F^{\prime}(E)-\lambda\right) V=0 .
$$

Thus, $\lambda=F^{\prime}(E)-k^{2} \pi^{2}$ for $k \in \mathbb{N}$ when $F^{\prime}(E)$ is constant or more generally $\left|F^{\prime}(E)-\lambda I\right|=0$.

Therefore, the operator $L$ is invertible if $F^{\prime}(E) \neq k^{2} \pi^{2}, \forall k \in \mathbb{N}$ or more generally when the eigenvalues of the Jacobian matrix at $E$ are non zero.

The operator $L$ is linear, and continuous between two normed spaces, therefore it is bounded.

The bounded inverse theorem states that a bijective bounded linear operator from a Banach space to another one has a bounded inverse. Thus, when the operator $L$ is invertible, its inverse is bounded.

We conclude that the implicit function theorem can be applied to $A(\epsilon, U)$ if $F^{\prime}(E) \neq k^{2} \pi^{2}, \forall k \in \mathbb{N}$.

Corollary A.2. Given a polynomial $p(\tau, x)=x^{n}+a_{n-1}(\tau) x^{n-1}+\cdots+a_{1}(\tau) x+a_{0}(\tau)$ whose coefficients depend smoothly on a parameter $\tau$ such that at $\tau=0$ the number $x=x_{0}$ is a simple root of this polynomial, $p\left(0, x_{0}\right)=0$. Then for all $\tau$ sufficiently near 0 there exists a unique root $x(\tau)$ with $x(0)=x_{0}$ that depends smoothly on $\tau$.

Proof. Given that $p\left(0, x_{0}\right)=0$ we want to solve $p(\tau, x)=0$ for $x(\tau)$ with $x(0)=x_{0}$. The assertions are immediate from the implicit function theorem. Since $x(0)=x_{0}$ is a simple zero of $p(0, x)=0$, then $p(0, x)=\left(x-x_{0}\right) g(x)$ , where $g\left(x_{0}\right) \neq 0$. Thus the derivative $p_{x}\left(0, x_{0}\right) \neq 0$.

Theorem A.3. Given a square matrix $A(\tau)$ whose elements depend smoothly on a real parameter $\tau$, if $\lambda=\lambda_{0}$ is a simple eigenvalue at $\tau=0$, then for all $\tau$ near 0 there is a corresponding eigenvalue that depends smoothly on $\tau$.

Proof. The proof is immediate from Corollary A.2 applied to the characteristic polynomial.

In brief, this appendix is about the Implicit Function Theorem. We start by stating and proving Theorem A.1 that emerges from the Implicit Function Theorem and that is applied to a particular form of problems. Finally, we recall Corollary A.2 and Theorem A.3.

Acknowledgements. The authors would like to thank Dr. Serge Assaf for the valuable insights and enriching discussions about the inflammatory aspects of atherosclerosis.

\section{REFERENCES}

[1] D. Abraham and O. Distler, How does endothelial cell injury start? The role of endothelin in systemic sclerosis. Arthritis Res Ther. 9 (2007) S2.

[2] L. Ai and K. Vafai, A coupling model for macromolecule transport in a stenosed arterial wall. Int. J. Heat Mass Transfer 49 (2006) 1568-1591.

[3] M. Atif Khan Bulelzai, Mathematical Models for Atherosclerotic Plaque Evolution. Ph.D. thesis, University of Singh (2013).

[4] E. August, K.H. Parker and M. Barahona, A dynamical model of lipoprotein metabolism. Bull. Math. Biol. 69 (2007) $1233-1254$

[5] B. Bartlett, H.P. Ludewick, A. Misra, S. Lee and G. Dwivedi, Macrophages and T cells in atherosclerosis: a translational perspective. Am. J. Physiol. Heart Circ. Physiol. 317 (2019) H375-H386.

[6] A.M.W. Bartosch, R. Mathews and J.M. Tarbell, Biophys. J. 113 (2017) 101-108.

[7] D. Bayik, D. Tross, L.A. Haile, D. Verthelyi and D.M. Klinman, Regulation of the maturation of human monocytes into immunosuppressive macrophages. Blood Adv. 1 (2017) 2510-2519.

[8] V. Bezyaev, N. Sadekov and V. Volpert, A model of chronic inflammation in atherosclerosis. Math. Model. Biomed. 31 (2020) 04002.

[9] Y. Bi, J. Chen, F. Hu, J. Liu, M. Li and L. Zhao, M2 macrophages as a potential target for antiatherosclerosis treatment. Neural. Plast. 2019 (2019) 6724903. 
[10] M.L. Brophy, Y. Dong, H. Wu, H.N.A. Rahman, K. Song and H. Chen, Eating the dead to keep atherosclerosis at bay. Front. Cardiovasc. Med. (2017).

[11] P.D. Cabral, N.J. Hong and J.L. Garvin, Shear stress increases nitric oxide production in thick ascending limbs. Am. J. Physiol. Renal Physiol. 299 (2010) F1185-F1192.

[12] C.V. Carman and R. Martinelli, T lymphocyte-endothelial interactions: emerging understanding of trafficking and antigenspecific immunity. Front Immunol. 6 (2015).

[13] D.S. Celermajer, C.K. Chow, E. Marijon, N.M. Anstey and K.S. Woo, Cardiovascular disease in the developing world: prevalences, patterns, and the potential of early disease detection. J. Am. Coll. Cardiol. 60 (2012) 1207-1216.

[14] C.A. Cobbold, J.A. Sherratt and S.R.J. Maxwell, Lipoprotein oxidation and its significance for atherosclerosis: a mathematical approach. Bull. Math. Biol. 64 (2002) 65-95.

[15] N.C. Di Paolo and D.M. Shayakhmetov, Interleukin $1 \alpha$ and the inflammatory process. Nat. Immunol. 17 (2016) $906-913$.

[16] G.A. Duque and A. Descoteaux, Macrophage cytokines: involvement in immunity and infectious diseases. Front. Immunol. 5 (2014).

[17] N. El Khatib, S. Génieys, B. Kazmierczak and V. Volpert, Reaction-diffusion model of atherosclerosis development. J. Math. Biol. 65 (2012) 349-374.

[18] N. El Khatib, S. Génieys and V. Volpert, Atherosclerosis initiation modeled as an inflammatory process. Math. Model. Nat. Phenom. 2 (2007) 126-141.

[19] N. El Khatib, O. Kafi, A. Sequeira, S. Simakov, Yu. Vassilevski and V. Volpert, Mathematical modelling of atherosclerosis. Math. Model. Nat. Phenom. 14 (2019) 603.

[20] A.R. Fatkhullina, I.O. Peshkova and E.K. Koltsova, The role of cytokines in the development of atherosclerosis. Biochemistry (Mosc). 81 (2016) 1358-1370.

[21] S. Freigang, F. Ampenberger, A. Weiss, T.D. Kanneganti, Y. Iwakura, M. Hersberger and M. Kopf, Fatty acid-induced mitochondrial uncoupling elicits inflammasome-independent $I L-1 \alpha$ and sterile vascular inflammation in atherosclerosis. Nat. Immunol. 14 (2013) 1045-1053.

[22] T. Gerhardt and K. Ley, Monocyte trafficking across the vessel wall. Cardiovasc. Res. 107 (2015) 321-330.

[23] M. Ghim, Y. Mohamied and P.D. Weinberg, The role of tricellular junctions in the transport of macromolecules across endothelium. Cardiovasc. Eng. Tech. 12 (2021) 101-113.

[24] D.M. Greif, M. Kumar, J.K. Lighthouse, J. Hum, A. An, L. Ding, K. Red-Horse, F. Hernan Espinoza, L. Olson, S. Offermanns and M.A. Krasnow, Radial construction of an arterial wall. Dev. Cell. 23 (2012) 482-493.

[25] H.A.R. Hadi, C.S. Carr and J. Al Suwaidi, Endothelial dysfunction: cardiovascular risk factors, therapy, and outcome. Vascu. Health Risk Manag. 1 (2005) 183-198.

[26] W. Hao and A. Friedman, The LDL-HDL profile determines the risk of atherosclerosis: a mathematical model. Plos One $\mathbf{9}$ (2014).

[27] B.J. Hunt and K.M. Jurd, Endothelial cell activation. BMJ 316 (1998) 1328-1329.

[28] A. Ibragimov, C. McNeal, L. Ritter and J. Walton, A mathematical model of atherogenesis as an inflammatory response. Math. Med. Biol. 22 (2015) 305-333.

[29] F. Ito and T. Ito, High-density lipoprotein (HDL) triglyceride and oxidized HDL: new lipid biomarkers of lipoprotein-related atherosclerotic cardiovascular disease. Antioxidants (Basel) 9 (2020) 362.

[30] S. Kawashima and M. Yokoyama, Dysfunction of endothelial nitric oxide synthase and atherosclerosis. Arterioscl. Thromb. Vasc. Biol. 24 (2004) 998-1005.

[31] U. Laufs, V. La Fata, J. Plutzky and J.K. Liao, Upregulation of endothelial nitric oxide synthase by HMG CoA reductase inhibitors. Circulation 97 (1998) 1129-1135.

[32] W. Lee and W.C. Liles, Endothelial activation, dysfunction and permeability during severe infections. Curr. Opin. Hematol. 18 (2011) 191-196.

[33] E. Leiva, S. Wehinger, L. Guzmán and R. Orrego, Role of oxidized LDL in atherosclerosis. Ann. N. Y. Acad. Sci. DOI: $10.5772 / 59375(2015)$.

[34] B.D. Li and J.L. Mehta, Oxidized LDL, a critical factor in atherogenesis. Cardiovasc. Res. 68 (2005) $353-354$.

[35] J.K. Liao, Linking endothelial dysfunction with endothelial cell activation. J. Clin. Invest. 123 (2013) $540-541$.

[36] P. Libby, The molecular mechanisms of the thrombotic complications of atherosclerosis. J. Int. Med. 263 (2008) $517-527$.

[37] P. Libby, Interleukin-1 beta as a target for atherosclerosis therapy: biological basis of CANTOS and beyond. J. Am. Coll. Cardiol. 70 (2017) 2278-2289.

[38] P. Libby, K. Bornfeldt and A.R. Tall, Atherosclerosis: successes, surprises, and future challenges. Circ Res. 118 (2016) 531-534.

[39] P. Libby, J.E. Buring, L. Badimon, G.K. Hansson, J. Deanfield, M. Sommer Bittencourt, L. Tokgözoglu and E.F. Lewis, Atherosclerosis. Nat. Rev. Disease Primers 5 (2019).

[40] A.K. Lund, Oxidants and endothelial dysfunction. Compreh. Toxicol. 13 (2018) 252-281.

[41] P. Macke Consigny, Pathogenesis of atherosclerosis. AJR 164 (1995) 553-558.

[42] C. McKay, S. McKee, N. Mottram, T. Mulholland and S. Wilson, Towards a Model of Atherosclerosis. Strathclyde Mathematics Research Report (2005).

[43] D. Mozaffarian, E.J. Benjamin, A.S. Go, et al., Heart disease and stroke statistics 2016 update: a report from the American Heart Association. Circulation 133 (2016).

[44] H.N. Mozar, D.G. Bal and S.A. Farag, The natural history of atherosclerosis: an ecologic perspective. Atherosclerosis 82 (1990) 157-164. 
[45] M. Mudau, A. Genis, A. Lochner and H. Strijdom, Endothelial dysfunction: the early predictor of atherosclerosis. Cardiovasc. J. Afr. 23 (2012) 222-231.

[46] S. Mundi, M. Massaro, E. Scoditti, M.A. Carluccio, V.W.M. Van Hinsbergh, M.L. Iruela-Arispe and R. De Caterina, Endothelial permeability, LDL deposition, and cardiovascular risk factors - a review. ESC Cardiovasc. Res. 114 (2018) 35-52.

[47] M.T. Nguyen, S. Fernando, N. Schwarz, J. TM Tan, C.A. Bursill and P.J. Psaltis, Inflammation as a therapeutic target in atherosclerosis. J. Clin. Med. 8 (2019) 1109.

[48] A. Ougrinovskaia, R. Thompson and M. Myerscough, An ODE model of early stages of atherosclerosis: mechanisms of the inflammatory response. Bull. Math. Biol. 72 (2010) 1534-1561.

[49] A.V. Panfilov, H. Dierckx and V. Volpert, (INVITED) Reaction-diffusion waves in cardiovascular diseases. Physica D 399 (2019) 1-34.

[50] R. Ross, Atherosclerosis: an inflammatory disease. N. Engl. J. Med. 340 (1999) 115-126.

[51] T. Silva, W. Jäger, M. Neuss-Raduc and A. Sequeira, Modeling of the early stage of atherosclerosis with emphasis on the regulation of the endothelial permeability. J. Theor. Biol. 496 (2020).

[52] I. Tabas and A.H. Lichtman, Monocyte-macrophages and T cells in atherosclerosis. Immunity 47 (2017) 621-634.

[53] S.S. Thosar, B.D. Johnson, J.D. Johnston and J.P. Wallace, Sitting and endothelial dysfunction: the role of shear stress. Med. Sci. Monit. 18 (2012) 173-180.

[54] D. Tousoulis, A.M. Kampoli, C. Tentolouris, N. Papageorgiou and C. Stefanadis, The role of nitric oxide on endothelial function. Curr. Vasc. Pharmacol. 10 (2012) 4-18.

[55] A. Tran-Dinh, D. Diallo, S. Delbosc, L.M. Varela-Perez, Q.B. Dang, B. Lapergue, E. Burillo, J.B. Michel, A. Levoye, J.L. Martin-Ventura and O. Meilhac, HDL and endothelial protection. Br. J. Pharmacol. 169 (2013) 493-511.

[56] V. Volpert, Elliptic Partial Differential Equations. Volume 2: Reaction-Diffusion equations. Birkhauser (2014).

[57] I. Wendelhag, O. Wiklund and J. Wikstrand, On quantifying plaque size and Intima-Media thickness in carotid and femoral arteries. Arterioscler. Thromb. Vasc. Biol. 16 (1996) 843-850.

\section{Subscribe to Open (S2O) A fair and sustainable open access model}

This journal is currently published in open access under a Subscribe-to-Open model (S2O). S2O is a transformative model that aims to move subscription journals to open access. Open access is the free, immediate, online availability of research articles combined with the rights to use these articles fully in the digital environment. We are thankful to our subscribers and sponsors for making it possible to publish this journal in open access, free of charge for authors.

\section{Please help to maintain this journal in open access!}

Check that your library subscribes to the journal, or make a personal donation to the S2O programme, by contacting subscribers@edpsciences.org

More information, including a list of sponsors and a financial transparency report, available at: https://www.edpsciences.org/en/maths-s2o-programme 